\title{
Flow Diagnosis and Optimization Based on Vorticity Dynamics for Transonic Compressor/Fan Rotor
}

\author{
Huanlong Chen', Mark G. Turner², Kiran Siddappaji², Syed Moez Hussain Mahmood² \\ ${ }^{1}$ Institute of Propulsion Theory and Technique, Harbin Institute of Technology, Harbin, China \\ ${ }^{2}$ Aerospace Engineering Department, University of Cincinnati, Cincinnati, OH, USA \\ Email:chenhuanlong@126.com,mark.turner@uc.edu
}

How to cite this paper: Chen, H.L., Turner, M.G., Siddappaji, K. and Mahmood, S.M.H. (2017) Flow Diagnosis and Optimization Based on Vorticity Dynamics for Transonic Compressor/Fan Rotor. Open Journal of Fluid Dynamics, 7, 40-71. https://doi.org/10.4236/ojfd.2017.71004

Received: December 19, 2016

Accepted: January 22, 2017

Published: January 25, 2017

Copyright $\odot 2017$ by authors and Scientific Research Publishing Inc. This work is licensed under the Creative Commons Attribution International License (CC BY 4.0). http://creativecommons.org/licenses/by/4.0/

\section{Open Access}

\begin{abstract}
This paper presents two optimized rotors. The first rotor is as part of a 3blade row optimization (IGV-rotor-stator) of a high-pressure compressor. It is based on modifying blade angles and advanced control of curvature of the airfoil camber line. The effects of these advanced blade techniques on the performance of the transonic 1.5-stage compressor were calculated using a 3D Navier-Stokes solver combined with a vortex/vorticity dynamics diagnosis method. The first optimized rotor produces a 3-blade row efficiency improvement over the baseline of $1.45 \%$ while also improving stall margin. The throttling range of the compressor is expanded largely because the shock in the rotor tip area is further downstream than that in the baseline case at the operating point. Additionally, optimizing the 3-blade row block while only adjusting the rotor geometry ensures good matching of flow angles allowing the compressor to have more range. The flow diagnostics of the rotor blade based on vortex/vorticity dynamics indicate that the boundary-layer separation behind the shock is verified by on-wall signatures of vorticity and skinfriction vector lines. In addition, azimuthal vorticity and boundary vorticity flux (BVF) are shown to be two vital flow parameters of compressor aerodynamic performance that directly relate to the improved performance of the optimized transonic compressor blade. A second rotor-only optimization is also presented for a 2.9 pressure ratio transonic fan. The objective function is the axial moment based on the BVF. An $88.5 \%$ efficiency rotor is produced.
\end{abstract}

\section{Keywords}

Transonic Compressor, Control Curvature Optimization, Vorticity Dynamics, BVF, Azimuthal Vorticity 


\section{Introduction}

The development of modern high thrust-weight ratio gas turbine engines for aircraft requires a compression system with high total pressure ratio and adiabatic efficiency, but also needs sufficient stall margin for stable and robust operation. If the blade loading for a fan/compressor is increased remarkably in one stage, the Mach Number and pressure gradient may be raised excessively leading to high diffusion and large scale boundary-layer separation may occur due to the shock wave or low momentum fluid accumulation in the corner. Thus the performance of a compressor on or off-design point will not be maintained. An advanced compressor aerodynamic optimization design technique and flow diagnosis method that can modify geometry parametrically, and take into account complex flow physics such as viscous shear, shocks, and vortex structure will aid further development of these compression systems.

Based on this motivation, many advanced aerodynamic design concepts and design system have been developed. In the early 1960s, Wang Zhongqi et al. [1] [2] [3] [4] proposed the concept and design method of the bowed/lean blade based on the theory of boundary-layer low-energy fluid radial migration. The bowed/lean blade technology with its ability to control end-wall boundary-layer migration has been widely applied to stator and rotor design. The initial attempted to apply the swept blade technique to design quiet high speed fan QF-12 to reduce fan noise in the NASA-Lewis Center between 1974 and 1977 [5] [6]. Wennerstrom introduced swept design concepts into the high-loaded transonic fan in the HTFC program [7]. The Air Force Research Labs at Wright-Patterson Air Force Base designed and tested a series of rotors to investigate the effects of swept blade [8] [9].

The early studies did not have 3D CFD to help in the design, and required extensive experiments to verify design concepts. More recently, optimization strategies that automate geometry creation with CFD simulation have been developed. Benini [10] and Jang et al. [11] researched the aerodynamic optimization design techniques based on the NASA Rotor 37 rotor. Three-dimensional viscous flow effects were taken into account through the 3D CFD approach. It showed that the shock wave structure and flow separation size were controlled by modifying the blade geometry. Lian et al. [12] used a multi-objective genetic algorithm examined blade aerodynamic optimization design for NASA Rotor 67 Rotor, and demonstrated not only the total pressure ratio and adiabatic efficiency for the rotor are improved, but also the boundary-layer depends on the blade section geometry variation on the transonic rotor surface, especially in the rotor tip area. Considering how to maintain a minimum stall margin up to $10 \%-15 \%$ for high-loading and high-through flow fan/compressor, Ellbrant et al. [13] presented optimization with a trade-off between stall margin (by optimizing pressure coefficient) at low speed and efficiency at high speed based on an automatic multi-objective optimization method. The results demonstrated a tradeoff between optimal efficiency and stall margin that could be weighed from a system perspective. 
A turbomachinery aerodynamic optimization design system based on smooth spanwise control of geometry [14] and controlling the curvature of the blade geometry [15] has been demonstrated by coupling a CFD solver to optimize efficiency [16]. Multi-objective optimization was also demonstrated by coupling to stress analysis to minimize weight as well as optimizing pressure coefficient at part speed similar to Ellbrant et al. [13]. A notable feature for this optimization system is that the aerodynamic performance is linked to the curvature distribution of airfoil camber line at several spanwise locations.

Vorticity and vortex structures appear inevitably in compressor viscous flows and heavily influence the aerodynamic performance of the compressor. Therefore the vorticity/vortex dynamics approach can be used to help guide and understand aerodynamic design and flow diagnosis for turbomachinery. It plays a significant role in turbomachinery aerothermodynamics theory and application [17] [18]. Additionally, vorticity dynamics is relevant to understand important physical concepts and characteristic parameters such as the boundary vorticity flux (BVF), vorticity vector, skin-friction vector, and azimuthal vorticity can be used in turbomachinery design and a flow analysis system. This can then provide some new analytical parameters, insight, and novel ideas for designers [19] [20] [21].

Optimization in the design process of fan/compressors has still not been utilized to its fullest potential. This is largely due to the large design space as well as the issues related to effectively defining actual design processes and precisely stating the optimization statement. Hence, the optimization platform defined by Nemnem et al. [16] is expanded for a 1.5-stage compressor focusing on modifying the rotor while taking into account the stationary blade rows on either side. This paper will describe the geometry parameters to be modified as well as the optimization process. The flow physics of the resulting optimized design will then be described. Moreover, the relation between the flow parameters and aerodynamic loading redistribution is explored using vorticity/vortex dynamics. This allows for examining the boundary-layer fluid accumulation and separation, as well as the shock wave boundary layer interaction. The results and methodology provides additional insight and can be helpful to designers of similar turbomachinery components.

\section{Pressure Gradient and Curvature}

The essential task of turbomachinery design is to create the geometry so the flow has minimum loss, with proper changes in flow direction or turning while ensuring the stress does not exceed strength and material properties of the structure. In general, a smooth aerodynamic shape is required for most of turbomachinery flow paths. In other words, the geometry curve should have continuous first and second derivatives. Mathematically, the curvature is defined as for a two-dimensional curve $y=f(x)$, It is also the reciprocal of the radius of curvature on the curve. Based on this formula, if the first and second derivatives are continuous, then the curvature is continuous. The crucial part is the second derivative 
$y$ " in the expression of curvature. Hence the second derivative is used for the airfoil parameters set as described by Nemnem et al. [15].

To illustrate the relation between the streamline curvature and static pressure gradient, an analysis of a fluid microelement unit in a blade to blade section is used, as shown in Figure 1. According to Newton's law, in a coordinate system tangential and normal to the streamline, the resulting equations are shown in Figure 1 (assuming inviscid flow) [22] [23]. The static pressure gradient normal to the streamline is balanced by the density times velocity squared divided by the streamline radius of curvature, $R$. It shows that it is vital to skillfully control streamline curvature in a design process, since pressure gradient of the fluid is influenced significantly by streamline curvature. As a designer of turbomachinery aerodynamics, controlling the curvature of geometry gives direct control of pressure gradients. Using curvature as the basis of controlling the camber line is therefore related to the flow physics.

Currently, many parametric curves such as cubic splines, B-splines and Bezier splines have been used to build three-dimensional blade. However, mathematically, the third derivative is not continuous on the node for these spline curves. Therefore, in order to avoid these problems, the cubic B-spline is applied to define the second derivative of the airfoil camber line. It is integrated twice to get points that define the camber line, which is then fourth derivative continuous. A smooth thickness distribution is then applied along with a smooth leading and trailing to generate the airfoil.

\section{Blade Aerodynamic Optimization System}

The turbomachinery aerodynamic optimization design system used to produce results in this paper is based on Shell script, Python scripts and Fortran executable in Linux framework. There are three main parts of it: the user-defined module, optimization script module and the Dakota optimization module [24] as shown in Figure 2. The user-defined module is to complete the definition for

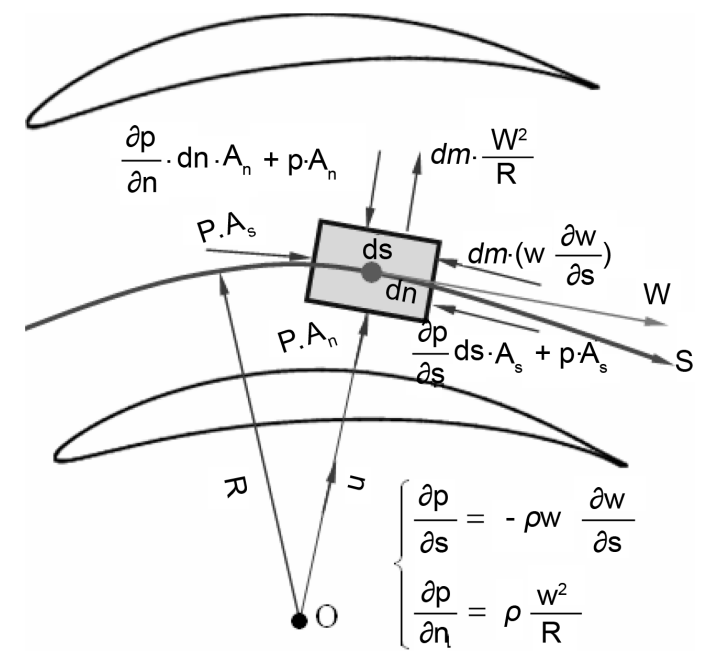

Figure 1. Force analysis for fluid microelement and resulting equations [22] [23]. 


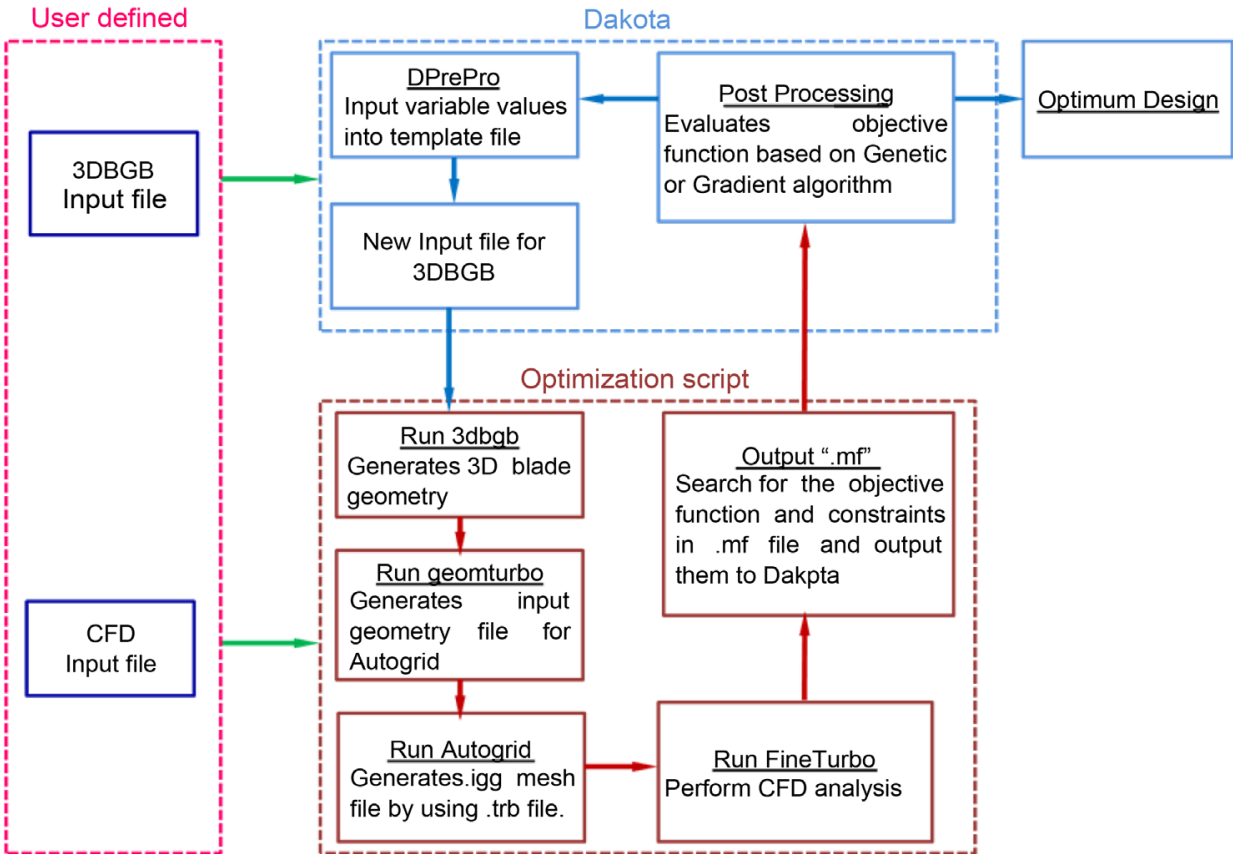

Figure 2. Optimization schematic.

optimization variables, parametric geometry, journal files for CFD solver, and mesh generation setting et al. The optimization script module automatically executes the three-dimensional blade geometry builder (3D BGB) [25] code used to generate $3 \mathrm{D}$ blade geometry and creates the geometry input file ${ }^{*}$.geomTurbo for Autogrid [26]. Autogrid is the mesh generator for the CFD solver Fine Turbo, which is run and output files are parsed to automatically obtain the objective function after each iteration of the optimization process. Dakota is used to guide the optimization process. For the results in this paper, a single objective function genetic algorithm (SOGA) is used, although Dakota has many more options for optimization.

The blade geometry generator (3D BGB) has many options to vary the $3 \mathrm{D}$ blade including smooth spanwise variations of leading and trailing edge angles, blade meanline and thickness, lean and sweep of the stacking axis, and chord. In the optimization of the first rotor, only blade leading and trailing edge angles and the blade meanline shape spanwise has been varied. By not varying the blade stacking, axial chord and stacking, it was felt that a stress analysis would not have to be done as part of a multiobjective optimization. The optimization could be used for aerodynamics only. This means there is a larger design space could be explored in the future, but with inherently more complexity.

The flow is not uniform at inlet and outlet of each blade row. The spanwise non-uniformity must be accounted for at the very least. A 1.5 stage simulation is therefore carried out to ensure the performance of the rotor is maintained within the multistage environment.

\section{Optimization of a Transonic Rotor}

From the mid-1970s to the early 1980s, NASA worked with industry Energy Ef- 
ficient Engine $\left(E^{3}\right)$ research program. One industry partner was GE, and the program advanced the design technology for all components that had an impact on engine efficiency. The core compressor that was designed was highly loaded with 10 stages and had a pressure ratio of 22.6. Holloway et al. [27] describe the design details of this compressor including the geometry of leading and trailing edge coordinates in the axial and radial direction as well as the blade angles. This information is also available as part of the 3D BGB [25] distribution. Further iterations of this compressor design led directly to the core compressor of the GE90 turbofan engine and several of its derivatives. Although the E3 core compressor by GE was highly successful, further improvements of core compression technology are highly desirable.

Accordingly, in order to illustrate the advantage of the optimization design and flow diagnosis method in this paper, the IGV and first stage of the $\mathrm{E}^{3}$ core compressor from GE has been chosen as the baseline case, and the rotor is optimized and analyzed in detail. Table 1 shows the main aerodynamic design parameters of the first stage rotor of the $\mathrm{GE}^{3}$ compressor. The baseline design uses $\mathrm{GE}^{3}$ axial and radial coordinates at the leading edge and trailing edge as well as the metal angles as defined in the report [27]. The flow path at hub and casing connects the leading and trailing edge points. A baseline curvature distribution, leading edge shape, and thickness available in 3D BGB [25] are used to construct the blade sections. This is not the GE $\mathrm{E}^{3}$ geometry, but one base on it. The modifications to the geometry for optimization will be the leading and trailing edge angles as well as modifying the curvature of the meanline. These adjustments have been made sequentially, first angle modification, and then curvature modifications explained in more detail in the next two subsections.

The quadrilateral meshes on the blade surfaces for 1.5-stage compressor is shown in Figure 3. The CFD simulations assume steady flow in a relative frame of reference. The relative frame of reference for a rotating domain in this paper has a constant angular velocity, and the relative flow equations are solved in this frame of reference. The S-A turbulence model is used with a mixing plane between each blade row. Gridding topology is a best practice established in Autogrid.

Table 1. Parameters of first stage of the GE $\mathrm{E}^{3}$ compressor [27].

\begin{tabular}{cc} 
Parameter & Value \\
\hline Corrected first rotor tip tangent speed $(\mathrm{m} / \mathrm{s})$ & 451.7 \\
Corrected design angular velocity $(\mathrm{rpm})$ & 12303 \\
Design mass flow rate $(\mathrm{kg} / \mathrm{s})$ & 54.4 \\
Tip flow coefficient of the first stage & 0.417 \\
Tip work coefficient of the first stage & 0.307 \\
Relative Mach Number at L.E. of the first rotor tip & 1.356 \\
Hub-to-tip ratio at the L.E. of the first rotor & 0.507 \\
The number of blades for IGV, Rotor 1 and Stator 1 & $32,28,50$ \\
Clearance at Rotor 1 tip (mm) & 0.5 \\
\hline
\end{tabular}




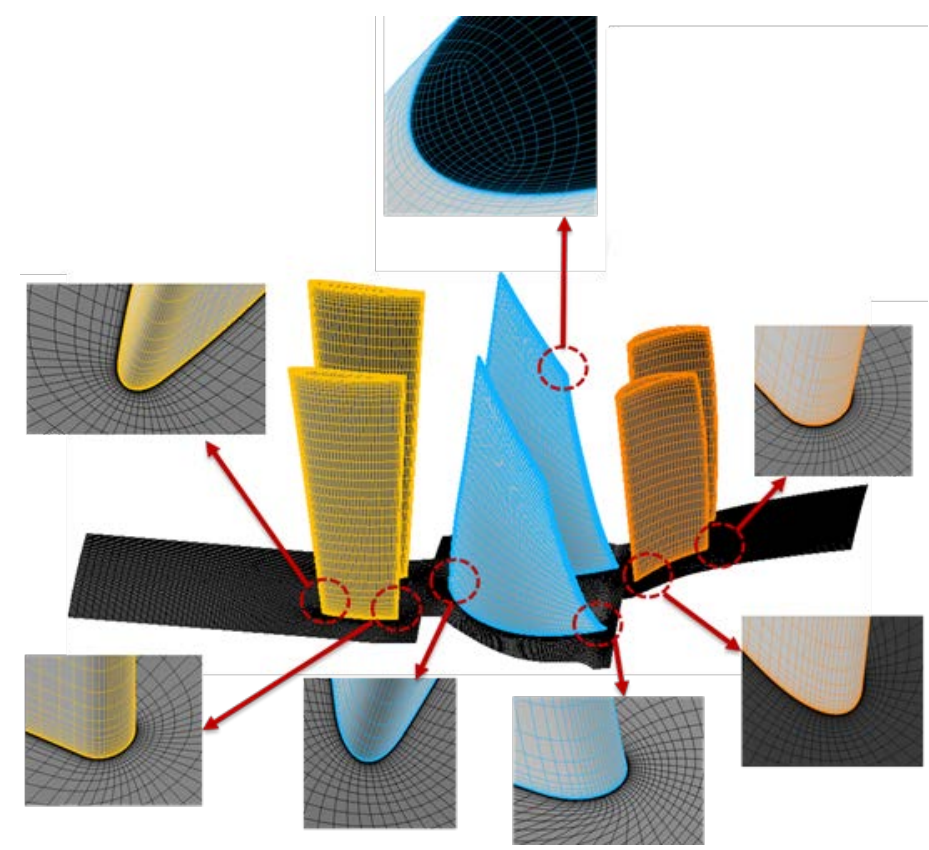

Figure 3. The meshes on the blade surfaces of the first 1.5 stages of the high pressure compressor.

$\mathrm{O}_{4} \mathrm{H}$ topological structure is applied to three blade rows, and the total hexahedron-mesh cell number is about 1,550,000 for 3 blade rows and the $y+$ of first grid cell is less than 1 in order to capture the flow details in boundary-layer area.

\subsection{Adjust Leading and Trailing Edge Blade Angles}

The design approach for high pressure compressors is to use a multi-fidelity approach starting with meanline, and then an axisymmetric solver with loss models which will give a starting point for a $3 \mathrm{D}$ blade shape. The $\mathrm{GE} \mathrm{E}^{3}$ compressor has been analyzed as described by Turner et al. [28] with an executable code that is freely available from the author on his website. Traditionally 3D CFD is used for each blade row in isolation except for the boundary conditions. Blade geometry and flow solutions would be tailored and run by hand.

Optimization has the potential to change this approach to design, although the designer must still remain integrally in the loop. This paper demonstrates an automated method of 3D CFD optimization process with parametric geometry manipulation. The genetic algorithm (GA) is effective for a relatively small number of design parameters. The GA approach is not as dependent on having fully converged CFD solutions, as is a gradient-based optimization method. The first optimization was performed to adjust the leading and trailing edge angles. The simulation domain is shown in Figure 4. Also shown in Figure 4 are the locations of the angle parameters $(\Delta \beta)$ that have been modified at the leading and trailing edges. These are adders to the original blade angles defined for the $\mathrm{E}^{3}$ compressor. These parameters modify a B-spline curve that ensures the modified blade is smooth. The IGV and stator 1 are the baseline and all other geometry parameters are kept the same. 


\section{Optimization Statement 1:}

Maximize: adiabatic efficiency $\eta$ for 1.5-stage domain;

Constraints: mass flux $\dot{m} \geq \dot{m}$ (baseline) total pressure ratio $\pi \geq \pi$ (baseline);

Design Variables: 10 variables, 5 control points for added flow angle $\Delta \beta$ at L.E. and 5 at the T.E. on rotor 1 as shown in Figure 4.

Genetic algorithm (GA) parameters: population size is 12 , and the maximum iteration is 100 .

Constants: All other parameters for blades are based on $\mathrm{E}^{3}$ report [27] and defaults.

The efficiency did not increase based on this optimization, but the mass flow increased by a small amount. The amount of angle change, $\Delta \beta$, at the rotor leading and trailing edge as a function of relative rotor blade height is shown in Figure 5. The figure demonstrates how the B-spline is defined by control points. The control points are modified through the optimization process to produce a smooth spanwise variation in the angle. Figure 5 indicates that the angle variation $\Delta \beta$ at the trailing edge is smaller than the leading edge at lower spanwise locations. The difference between these angles represents the net camber difference.

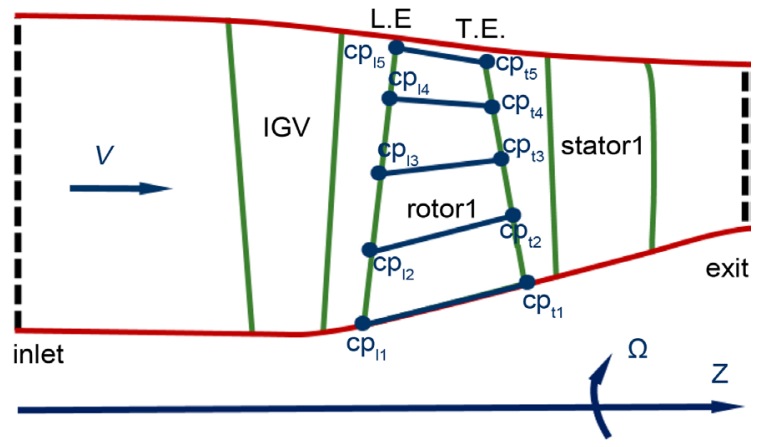

Figure 4. Solution domain for IGV and first stage for optimization. Also shown are the angle control points used for the first optimization on rotor 1.

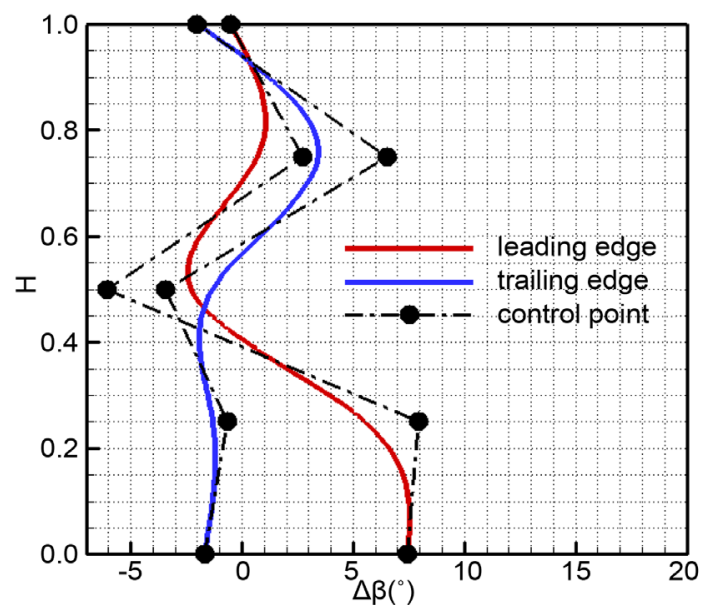

Figure 5. Flow angle adders at rotor L.E. and T.E. 


\subsection{Control Curvature of Rotor Camber Line}

The curvature of the airfoil camber line is modified by changing spanwise B-spline control points (similar to the angle adder shown in Figure 5) which define curvature at each span. Figure 6 shows the resulting curvature control values at 4 sections for the optimized blade. This approach is described by Nemnem et al. [15] and Mahmood et al. [29]. The curvature control points define a Bspline of the second derivative of the meanline. This curve is then integrated twice to obtain the meanline curve. At $75 \%$ span the sign of the second derivative changes twice. This implies that the blade meanline at that span has an s-shape.

Optimization Statement 2:

Maximize: adiabatic efficiency $\eta$ for 1.5 -stage domain;

Constraints: mass flux $\dot{m} \geq \dot{m}$ (baseline) total pressure ratio $\pi \geq \pi$ (baseline);

Design Variables: 24 variables for 4 sections, which have 3 inside points are used to control curvature for the unit chord as seen in Figure 6.

Genetic algorithm (GA) parameters: population size is 12 , and the maximum iteration is 70 .

Constants: Use all other blade definitions from the result of optimization 1.

A comparison of baseline and optimum blade shapes are shown in Figure 7
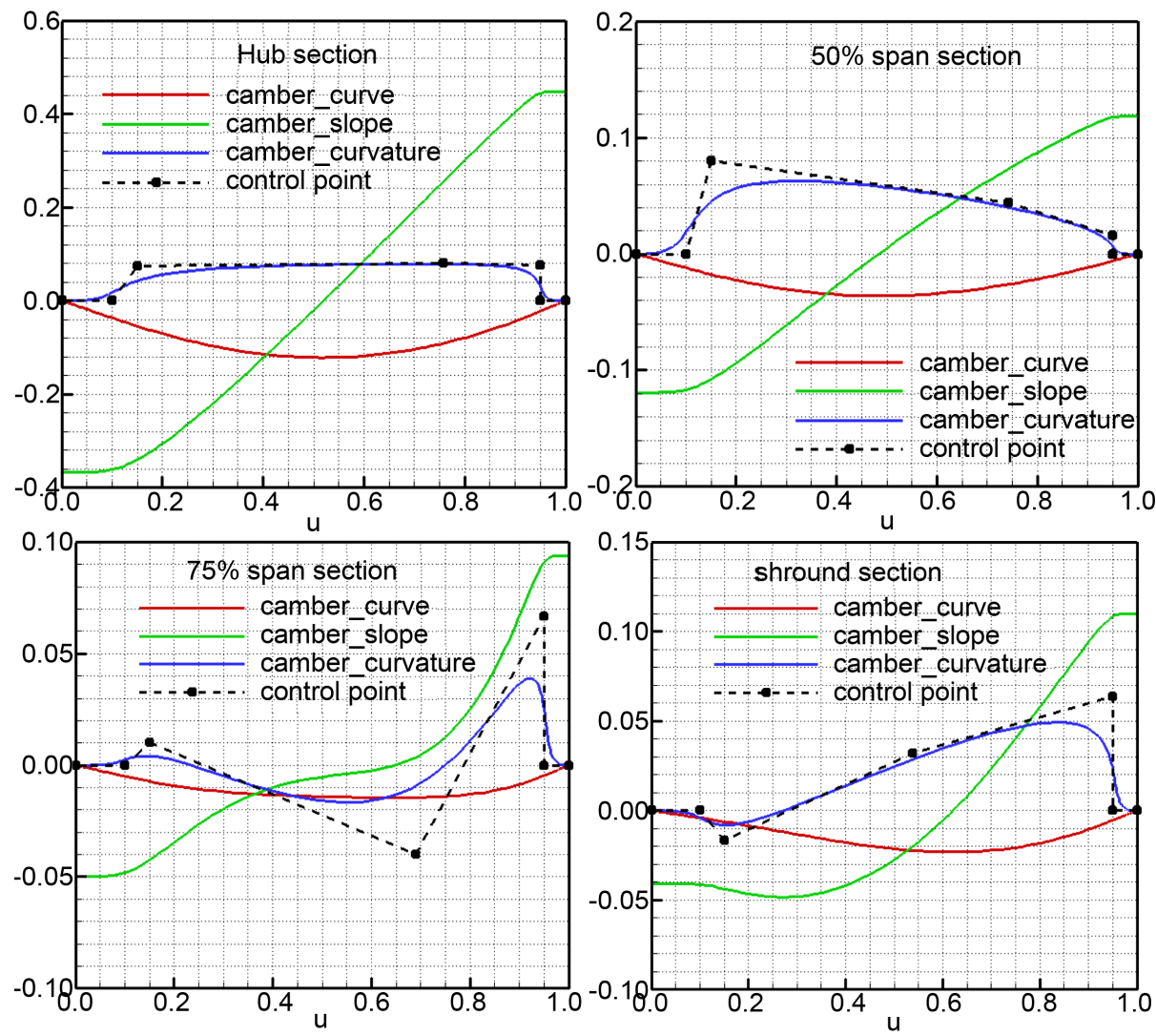

Figure 6. Camber line and its slope and curvature. The control points define the B-spline of the camber line second derivative. 
and Figure 8. This is the result of both optimizations 1 and 2, and for the rest of the paper, optimimum refers to the result of both steps.

The curvature control through the optimization allows for an indirect control of the pressure gradients. Figure 9 shows the overall compressor performance
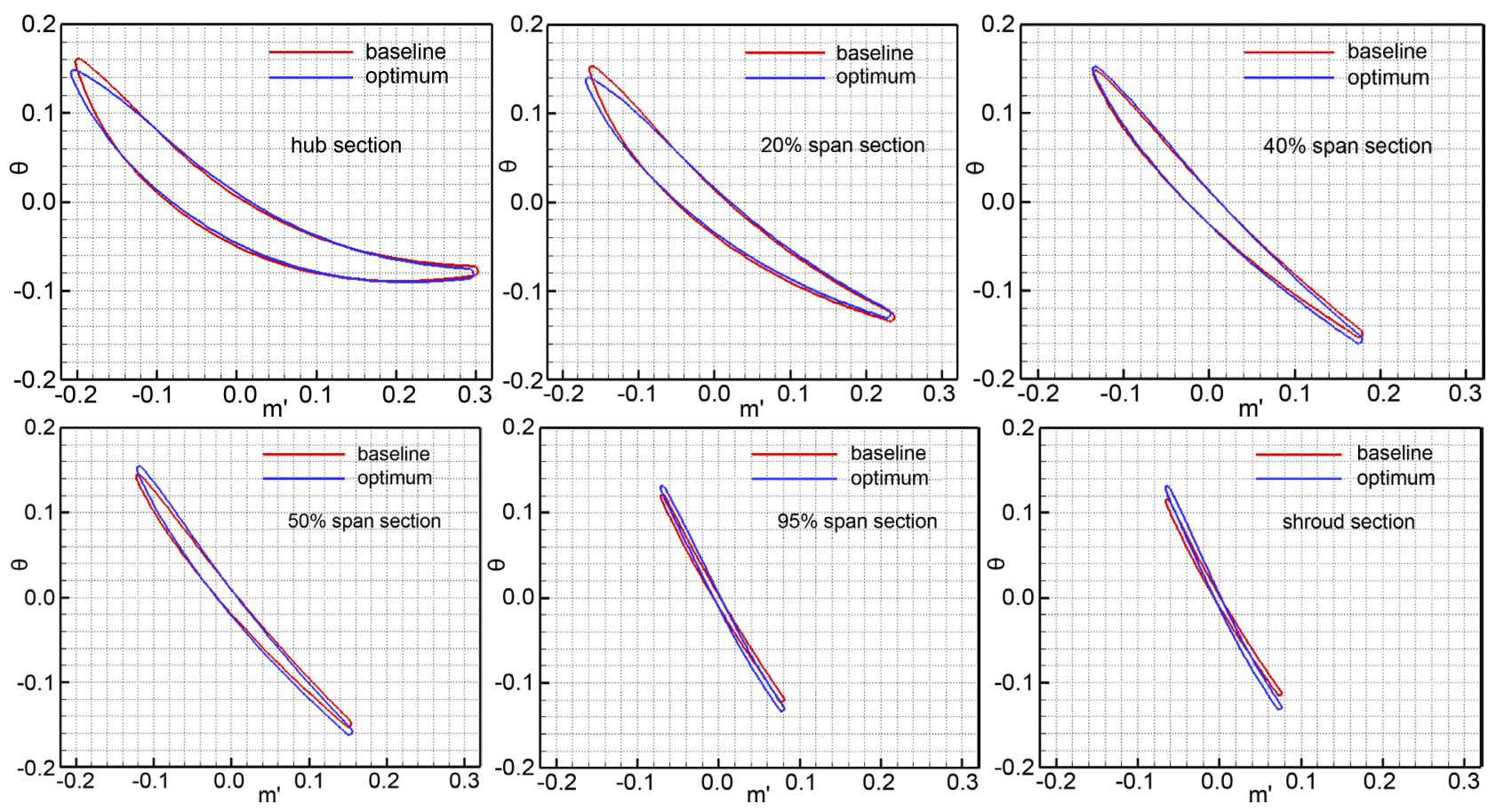

Figure 7. Blade profiles of baseline and optimum case.

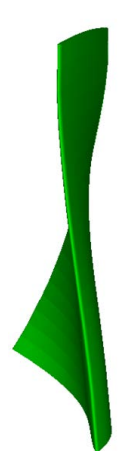

(a) View the 3D rotor from leading edge (green-baseline)

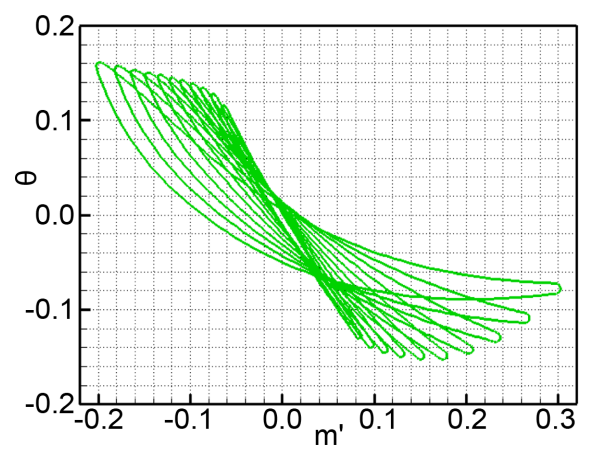

(c) Stacking airfoil on the centroid for baseline rotor

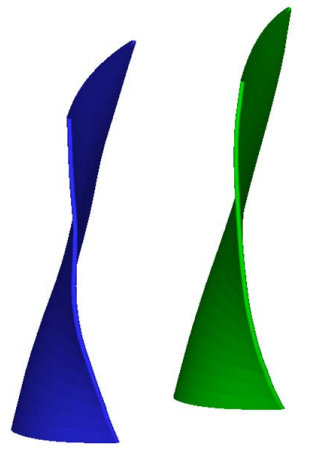

(b) View the 3D rotor from trailing edge (blue-optimum)

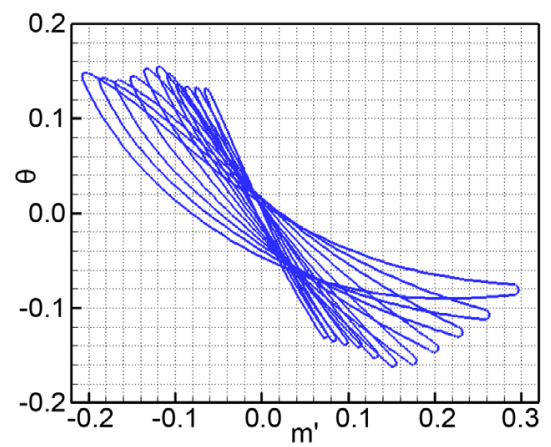

(d) Stacking airfoil on the centroid for optimum rotor

Figure 8. 3D geometry for rotor of baseline and optimum. 
for baseline, optimizations 1 and 2 on the design angular velocity. It demonstrates that the adiabatic efficiency, total pressure ratio and mass flow rate are increased. Table 2 summarizes the results.

These are overall parameters of the 3-blade row block, and having the rotor optimized with the stationary blade rows included allows for the correct matching of the flow field. The adiabatic efficiency of the 3-blade row block increases by $1.45 \%$ (delta efficiency), whereas the rotor-only efficiency gain is $1.27 \%$ (delta efficiency). The block efficiency gain is higher, implying there is improvement in the stationary blade rows due to better matching. The optimization constraint was to have a higher mass flux than the baseline. The optimum increased the mass flow by nearly $0.6 \%$ to $53.97 \mathrm{~kg} / \mathrm{s}$ for the design back pressure. This is still lower than the $\mathrm{E}^{3}$ design intent of $54.4 \mathrm{~kg} / \mathrm{s}$. The design report [27] states that the first test of the compressor which is also the basis of the geometry used here reached the design flow rate at $102 \%$ corrected speed. The baseline geometry is therefore consistent with this goal. Geometry changes for subsequent builds are discussed in the report, but not enough details are given to know the key features of that geometry. The total pressure ratio increases slightly by $0.223 \%$, while the stall mass flow rate is decreased by $1.541 \%$, thus the stall margin and the operation range are improved for the optimized case.

Figure 10 shows the adiabatic efficiency and total pressure ratio profiles as a function of relative blade height. It indicates that the total pressure ratio is

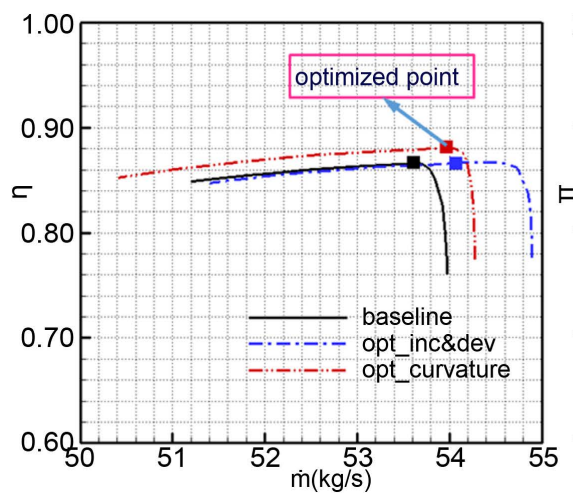

(a) The adiabatic efficiency vs. mass flow rate

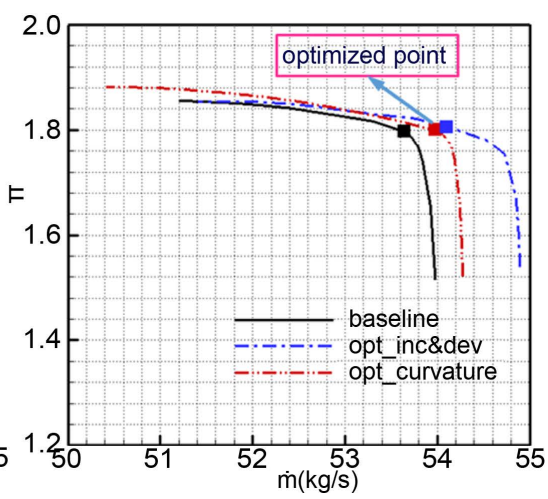

(b) The total pressure ratio vs. mass flow rate

Figure 9. Constant speed lines for 1.5-stage compressor.

Table 2. Compressor performance before and after optimization (with LE and TE angles plus curvature).

\begin{tabular}{ccccc}
\hline \multicolumn{2}{c}{ Parameters } & Baseline & Optimum & Percentage \\
\hline & Efficiency $(\eta)$ & $86.63 \%$ & $88.08 \%$ & $\mathbf{+ 1 . 6 7 \%}$ \\
$\begin{array}{c}\text { Optimized point } \\
\text { (3-blade rows) }\end{array}$ & $\operatorname{PR}(\pi)$ & 1.797 & 1.800 & $\mathbf{+ 0 . 2 2 3 \%}$ \\
Mass flow rate $(\mathrm{kg} / \mathrm{s})$ & 53.65 & 53.97 & $\mathbf{+ 0 . 5 9 6 \%}$ \\
Optimized point & Efficiency $(\eta)$ & $89.39 \%$ & $90.66 \%$ & $+\mathbf{1 . 4 2 \%}$ \\
(rotor-only) & $\operatorname{PR}(\pi)$ & 1.832 & 1.834 & $\mathbf{+ 0 . 1 0 9 \%}$ \\
\multicolumn{2}{c}{ Choking flow rate $(\mathrm{kg} / \mathrm{s})$} & 53.98 & 54.27 & $\mathbf{+ 0 . 5 3 7 \%}$ \\
\multicolumn{2}{c}{ Stall mass flow rate $(\mathrm{kg} / \mathrm{s})$} & 51.19 & 50.40 & $\mathbf{- 1 . 5 4 \%}$ \\
\hline
\end{tabular}


reduced slightly due to the flow turning angle being decreased below $40 \%$ span. By contrast it is raised at the outer regions of the span mainly due to the strength of shock wave being decreased as will be described in more detail. The adiabatic efficiency is improved over the entire span mainly due to the smooth variations of the curvature parameters and their link spanwise.

The link between the flow and blade geometry controlling curvature can be seen in the relative Mach Number at $95 \%$ span as shown in Figure 11. It demonstrates that not only the upstream Mach Number of the rotor leading edge shock wave is decreased, but also the position of shock wave is moved closer to the trailing edge for the optimum case. This is beneficial since there is a decreased
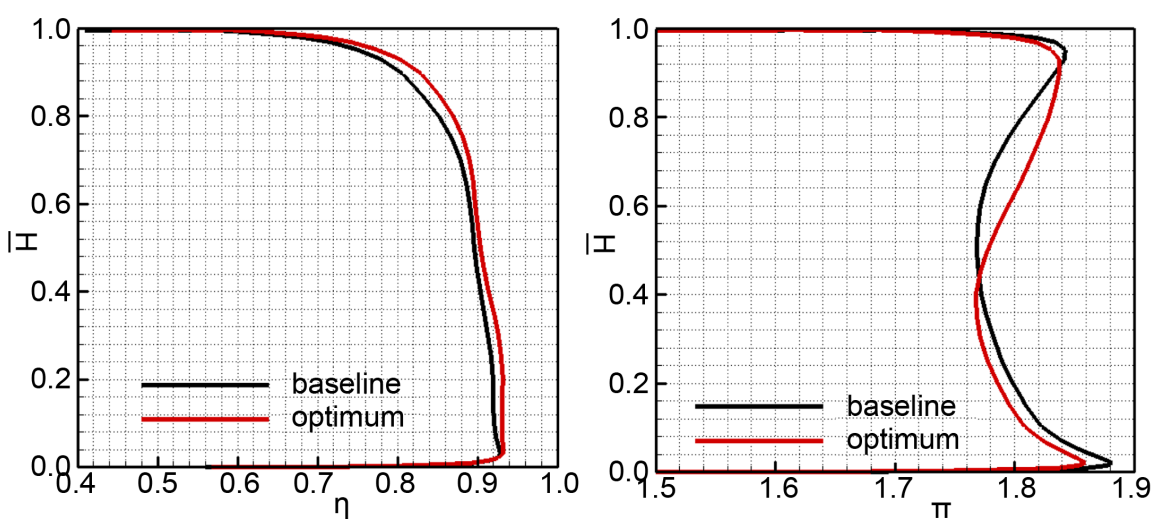

(a) The adiabatic efficiency vs. relative blade height (b) The total pressure ratio vs. relative blade height

Figure 10. Aerodynamic performance vs. relative blade height for compressor.

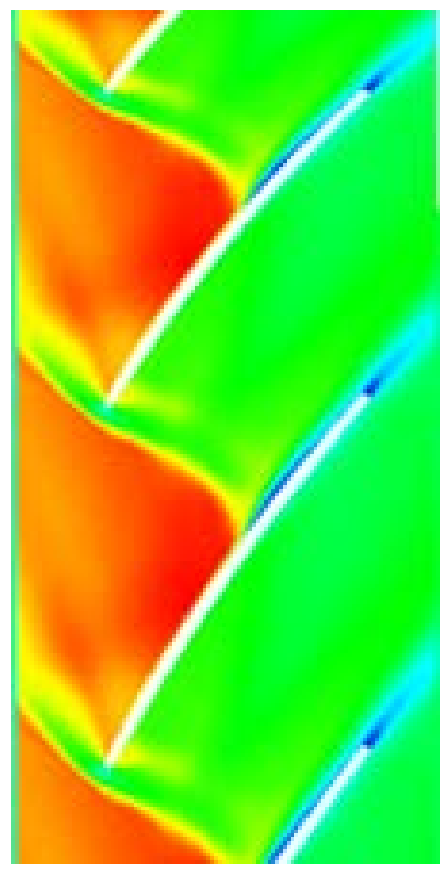

(a) Baseline case

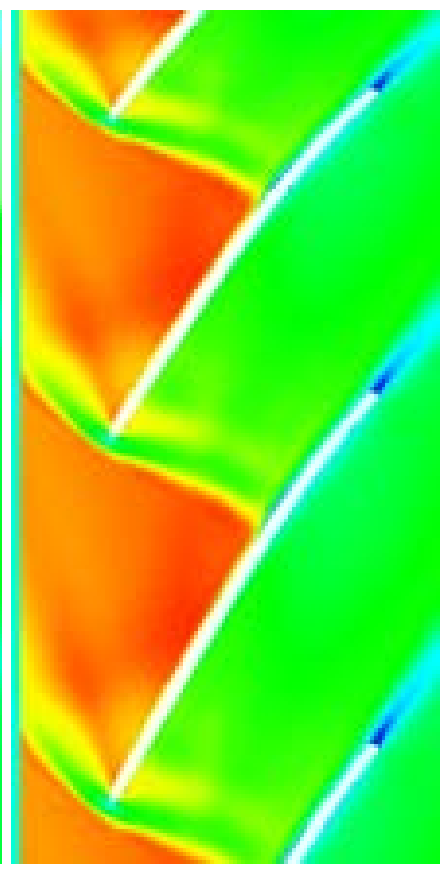

(b) Optimum case (1 \& 2)

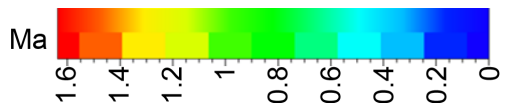

Figure 11. Mach Number for rotor at $95 \%$ span section. 

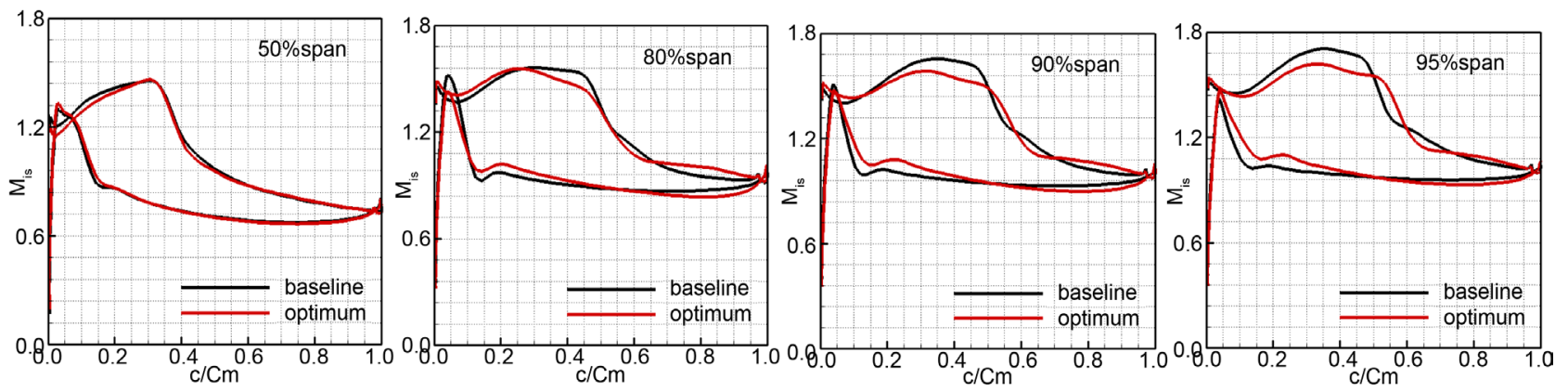

Figure 12. Isentropic Mach Number vs. meridian line for rotor of baseline and optimized control curvature case (1 \& 2).

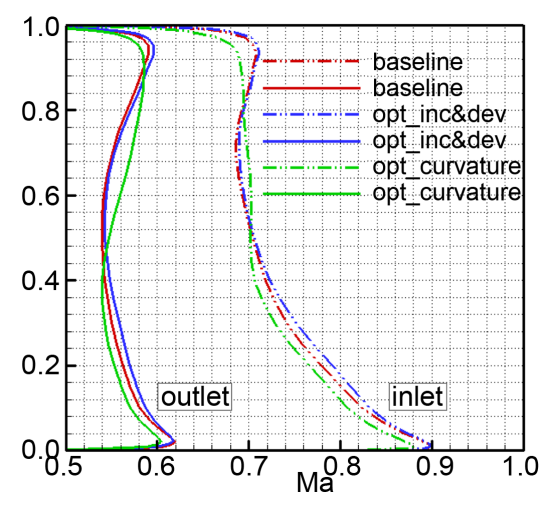

Figure 13. Absolute Mach Number at inlet and outlet for stator. 
ratio increases, the pressure gradient is appropriately modified and the whole aerodynamic performance of the compressor block is improved remarkably.

\section{Vorticity/Vortex Dynamics Diagnosis}

Vortex/vorticity dynamics is an important concept in fluid dynamics, which has played an exceptionally crucial role in turbomachinery. It provides novel ideas for turbomachinery aerodynamic design and viscous flow diagnosis by focusing on the derivative/gradient of flow parameters to examine essential flow information and underlying dynamic processes. In this section, the physical mechanisms for boundary-layer separation due to expansion or compression and shear effects, such as shock wave and corner separation are explored from the viewpoint of vorticity dynamics. The physical cause of improving performance of the compressor by controlling curvature will be analyzed in detail, and this relatively new flow diagnosis method for transonic compressors is presented.

The relationship between vorticity and throughflow capability of a compressor will be explored by deducing the expression for azimuthal vorticity $\omega_{\theta}$. The discussion is limited to a single axial-flow compressor rotor as shown in Figure 14. In the cylindrical coordinates system $(r, \theta, z)$, the mass flow rate through the $Z=$ $Z_{2}$ plane that is perpendicular to the $\mathrm{z}$-axis can be expressed as follows:

$$
\begin{aligned}
\int_{S} \rho u_{z} \mathrm{~d} S & =\int_{s} \rho u_{z} r \mathrm{~d} r \mathrm{~d} \theta=\frac{1}{2}\left[\left.\int_{0}^{2 \pi} \rho u_{z} r^{2}\right|_{r_{1}} ^{r_{2}} \mathrm{~d} \theta-\int_{S} r \frac{\partial \rho u_{z}}{\partial r} \mathrm{~d} S\right] \\
& =\left.\pi \rho u_{z} r^{2}\right|_{r_{1}} ^{r_{2}}-\frac{1}{2} \int_{s} r \frac{\partial \rho u_{z}}{\partial r} \mathrm{~d} S=-\frac{1}{2} \int_{s} r \frac{\partial \rho u_{z}}{\partial r} \mathrm{~d} S \\
& =-\frac{1}{2} \int_{s} r\left(u_{z} \frac{\partial \rho}{\partial r}+\rho \frac{\partial u_{z}}{\partial r}\right) \mathrm{d} S=-\frac{1}{2} \int_{s} r\left[u_{z} \frac{\partial \rho}{\partial r}+\rho\left(\frac{\partial u_{z}}{\partial r}-\omega_{\theta}\right)\right] \mathrm{d} S
\end{aligned}
$$

where, $r_{1}$ and $r_{2}$ are radii for hub and casing respectively. The axisymmetric assumption means $\rho u_{z}$ is a constant in the azimuthal direction. Moreover, $\partial / \partial z \ll \partial / \partial r$ in the outlet plane for axial-flow turbomachinery, so the azimuthal vorticity $\omega_{\theta}=\partial u_{r} / \partial z-\partial u_{z} / \partial r \approx-\partial u_{z} / \partial r$. According to the no-slip condition for a viscous fluid, $u_{z, r_{1}}=u_{z, r_{2}}=0$ for the hub and casing positions, and

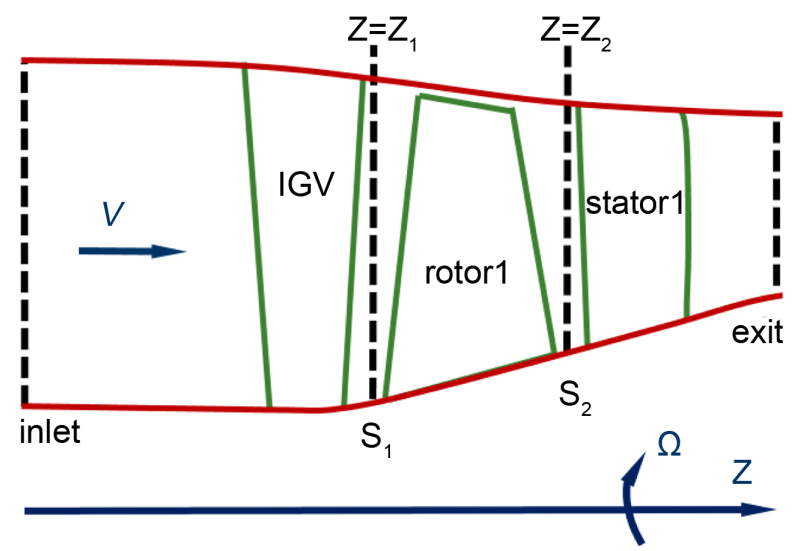

Figure 14. Solution domain showing constant axial lines used for analysis. 
$\frac{\partial \rho}{\partial r} \approx 0, \frac{\partial u_{r}}{\partial z} \approx 0$ in the outlet plane far from the trailing edge of blade for axial-flow turbomachinery. Therefore, the Equation (1) is simplified as follows:

$$
\int_{s} \rho u_{z} \mathrm{~d} S \approx \frac{1}{2} \int_{s} r \rho \omega_{\theta} \mathrm{d} S=\pi \int_{r_{1}}^{r_{2}} r^{2} \rho \omega_{\theta} \mathrm{d} r
$$

Considering the designers main focus on the meridonal flow parameters, an analysis of meridional azimuthal vorticity $\omega_{\theta}=\partial u_{r} / \partial z-\partial u_{z} / \partial r$ is shown in Figure 15. There are three zones in the meridional plane: the near-hub zone $A_{1}$, the near-casing zone $\mathrm{A}_{3}$ and the main flow core zone $\mathrm{A}_{2}$. In the $\mathrm{A}_{2}$ zone, $\omega_{\theta} \approx 0$ due to $\partial u_{r} / \partial z \approx \partial u_{z} / \partial r \approx 0$. In the $A_{1}$ zone, $\partial u_{r} / \partial z \ll \partial u_{z} / \partial r$, and $\partial u_{z} / \partial r>0$, so $\omega_{\theta} \approx-\partial u_{z} / \partial r<0$. However, in the $A_{3}$ zone, $\partial u_{r} / \partial z \ll \partial u_{z} / \partial r$, and $\partial u_{z} / \partial r<0$, so $\omega_{\theta} \approx-\partial u_{z} / \partial r>0$.

According to Equation (2) and the discussion of Figure 15, the mass flow rate of a compressor would be increased if there is an increase in the positive azimuthal vorticity $\omega_{\theta}$ in the near-casing zone $\mathrm{A}_{3}$ and make $\mathrm{A}_{3}$ with a higher radius. Alternatively a decrease of negative azimuthal vorticity $\omega_{\theta}$ in the near-hub zone $A_{1}$ and make $A_{1}$ with lower radius would also increase the mass flow rate. From a traditional fluid dynamics viewpoint, this means the same as reducing the boundary-layer thickness.

The discussion is explained further using Figure 16 and Figure 17 for azimuthal vorticity in a cross-section within the rotor (at $70 \%$ chord) and at the stator exit planes, respectively. The azimuthal vorticity distribution demonstrates that the higher $\omega_{\theta}$ area is smaller for the optimum case than for the baseline case, which mainly occurs in the near-casing and near-wall of suction side zone for rotor cross section, and in the near-casing and lower spanwise area for stator outlet plane. It illustrates the flow core zone is limited and the boundary-layer thickness is decreased partially for the optimum case compared to the baseline case. Therefore the mass flow rate, total pressure ratio and adiabatic efficiency are improved wholly for the optimum case as already shown in Figure 9 and Table 2 . This is also demonstrated with the axial vorticity $\omega_{z}$ as shown in Figure 18.

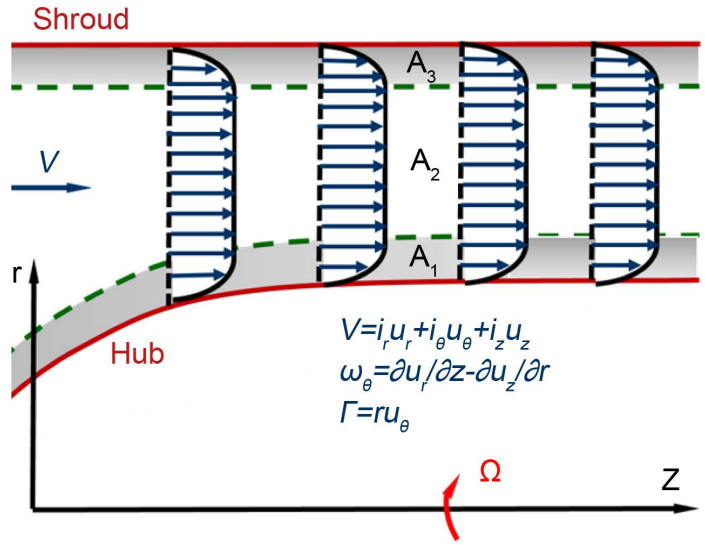

Figure 15. Sketch of meridional plane for azimuthal vorticity analysis. 


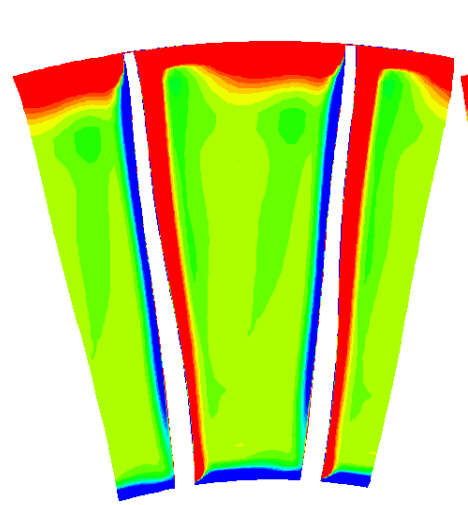

(a) Baseline case

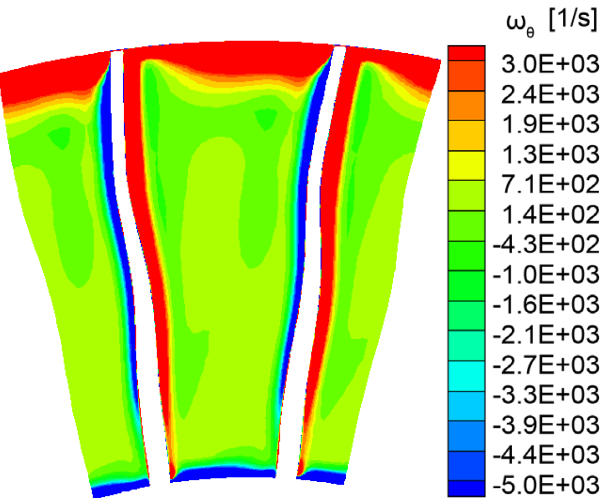

(b) Optimum case ( 1 \& 2)

Figure 16. Azimuthal vorticity in cross section in the rotor (at $70 \%$ chord).

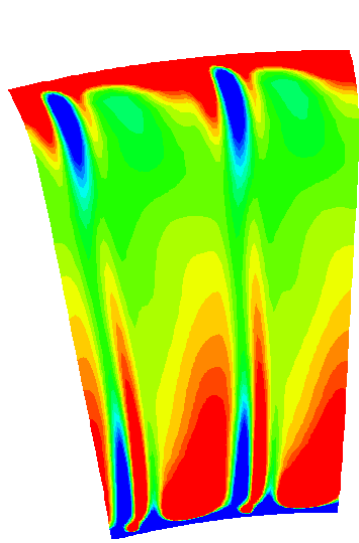

(a) Baseline case

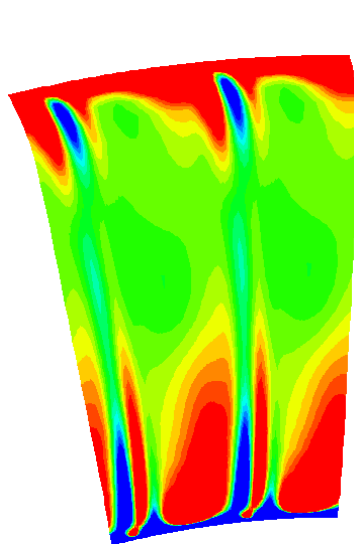

(b) Optimum case (1 \& 2) $\omega_{\theta}[1 / \mathrm{s}]$ $3.0 \mathrm{E}+03$ $2.4 \mathrm{E}+03$ $1.9 \mathrm{E}+03$ $1.3 \mathrm{E}+03$ 7.1E+02 $1.4 \mathrm{E}+02$ $-4.3 E+02$ $-1.0 \mathrm{E}+03$ $-1.6 \mathrm{E}+03$ $-2.1 \mathrm{E}+03$ $-2.7 \mathrm{E}+03$ $-3.3 E+03$ $-3.9 E+03$ $-4.4 \mathrm{E}+03$ $-5.0 \mathrm{E}+03$

Figure 17. Azimuthal vorticity in cross section at stator exit.

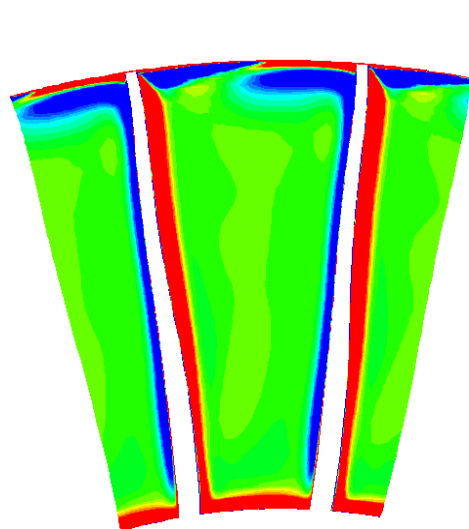

(a) Baseline case

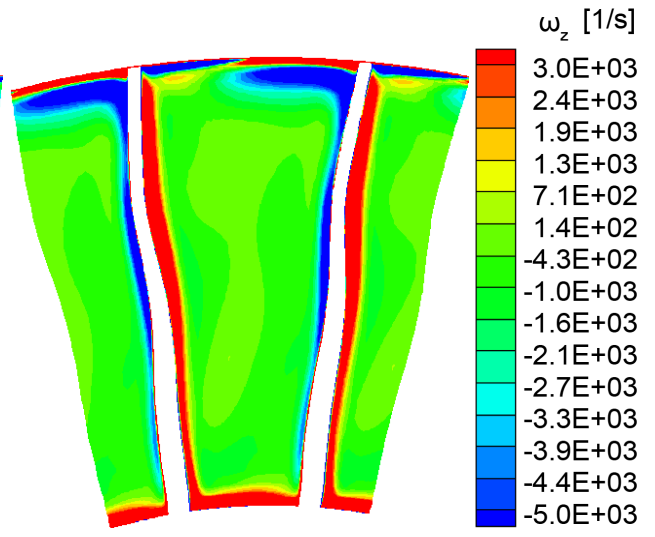

(b) Optimum case (1 \& 2)

Figure 18. Axial vorticity in a cross section in the rotor (at $70 \%$ chord).

The radial vorticity $\omega_{r}$ and skin-friction vector $\tau$ lines are shown in Figure 19 on the suction side of the rotor. The 3D view for Figure 19 and Figures 20-28 is shown as in Figure 29 by Tecplot [30]. It indicates that the separation flow area and the radial vorticity $\omega_{r}$ is decreased entirely for the optimum case compared to the baseline case. It illustrates that the shock strength and separated flow 


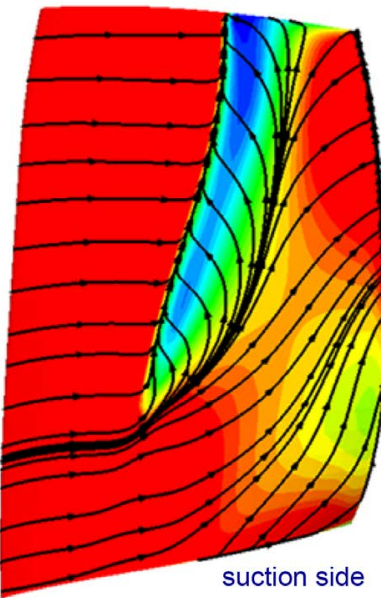

(a) Baseline case

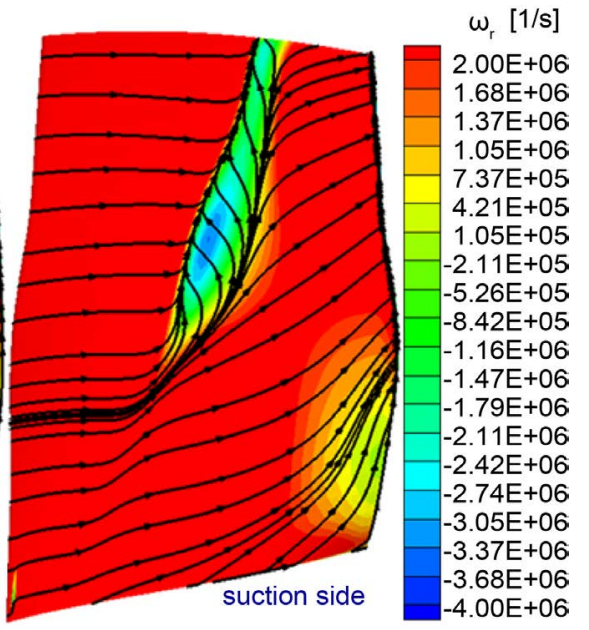

(b) Optimum case (1 \& 2)

Figure 19. Radial vorticity and skin-friction vector lines on rotor suction side.

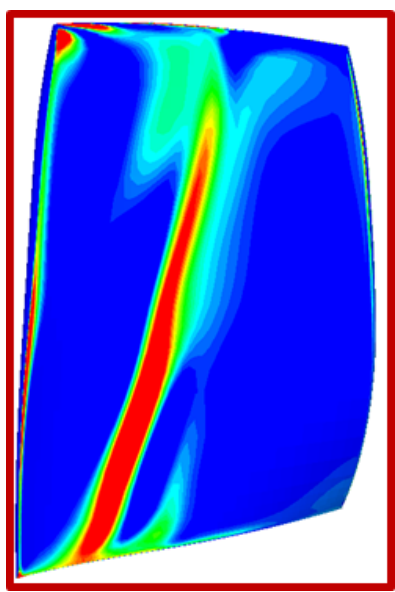

(a) Baseline case

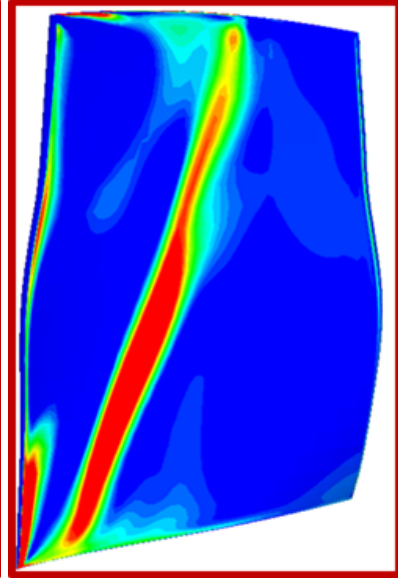

(b) Optimum case $(1 \& 2)$

$\sigma_{\mathrm{pz}}\left[\mathrm{m} / \mathrm{s}^{2}\right]$

$6.00 \mathrm{E}+05$ $5.67 \mathrm{E}+05$ $5.35 \mathrm{E}+05$

$5.02 \mathrm{E}+05$

$4.69 \mathrm{E}+05$

$4.37 \mathrm{E}+05$

$4.04 \mathrm{E}+05$

$3.72 \mathrm{E}+05$

$3.39 \mathrm{E}+05$

$3.06 \mathrm{E}+05$

$2.74 \mathrm{E}+05$

$2.41 \mathrm{E}+05$

$2.08 \mathrm{E}+05$

$1.76 \mathrm{E}+05$

$1.43 \mathrm{E}+05$

$1.11 \mathrm{E}+05$

$7.79 \mathrm{E}+04$

$4.53 \mathrm{E}+04$

$1.26 \mathrm{E}+04$

$-2.00 \mathrm{E}+04$

Figure 20. BVF- $\sigma_{p z}$ on rotor suction side.

Static pressure [pa]

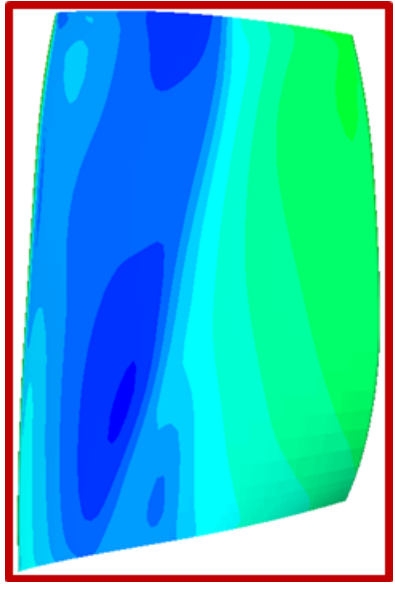

(a) Baseline case

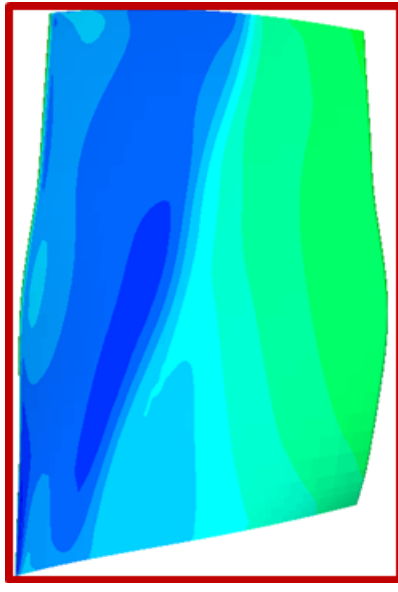

(b) Optimum case (1 \& 2)

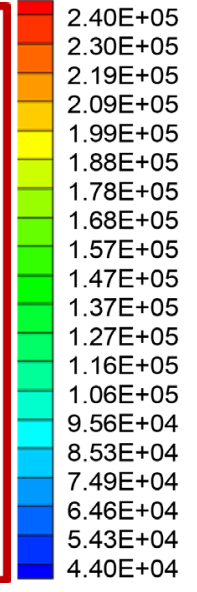

$4.40 \mathrm{E}+04$

Figure 21. Static pressure on rotor suction side. 


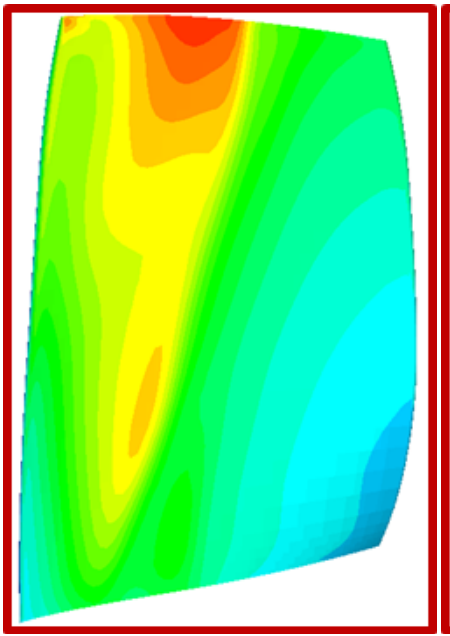

(a) Baseline case

Entropy $[\mathrm{J} /(\mathrm{kg} . \mathrm{k})]$

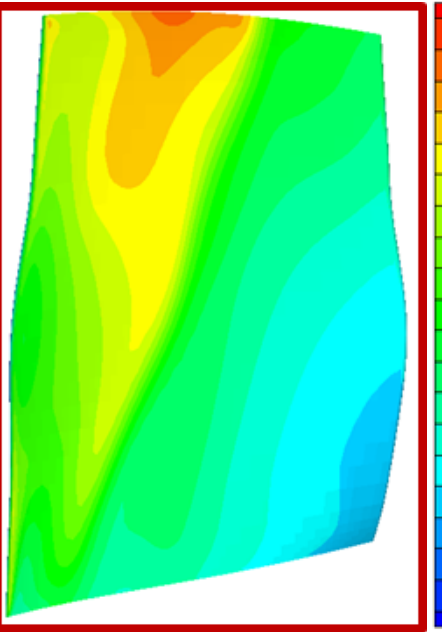

(b) Optimum case (1 \& 2)

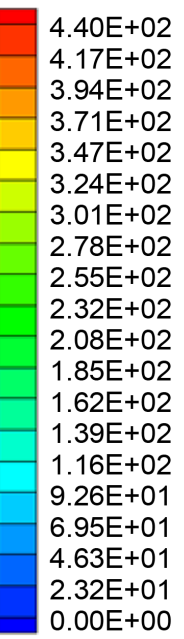

$0.00 \mathrm{E}+00$

\section{side.}

Figure 22. Entropy on rotor suction side.

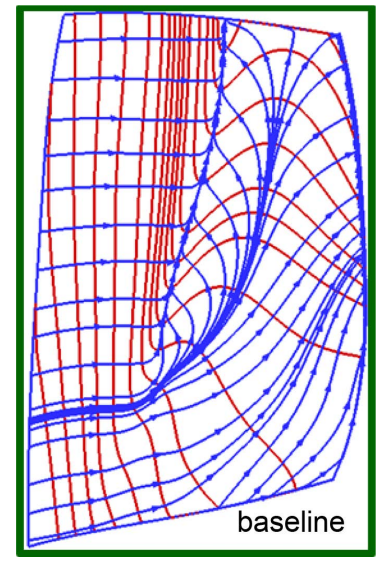

(a) Baseline case

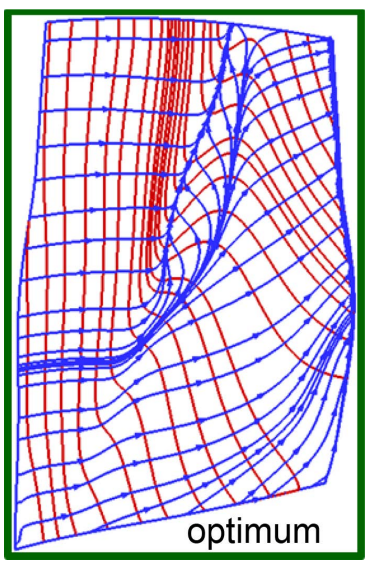

(b) Optimum case ( $1 \& 2)$

Figure 23. Vector lines of $(\boldsymbol{\tau}, \boldsymbol{\omega})$ on rotor suction side (red curve is $\omega$, blue curve is $\tau$ ).

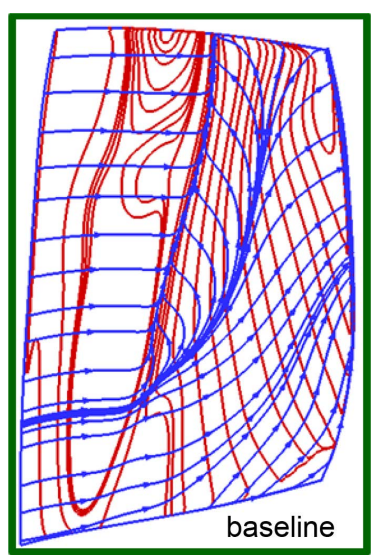

(a) Baseline case

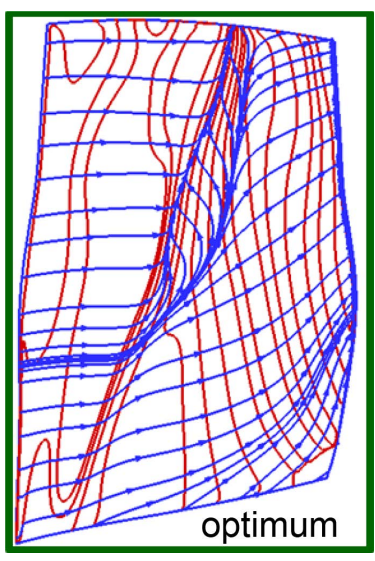

(b) Optimum case (1 \& 2)

Figure 24. Vector lines of $\left(\tau, \sigma_{p}\right)$ on rotor suction side (red curve is $\sigma_{p}$, blue curve is $\tau$ ). 


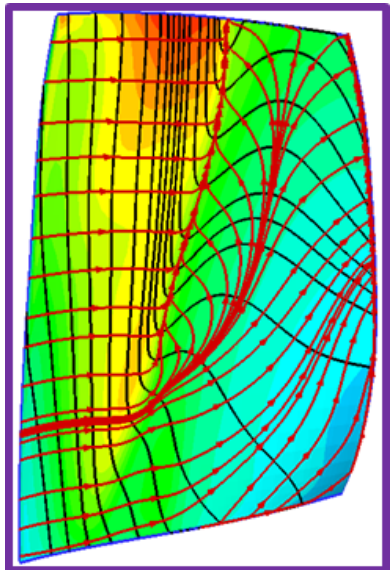

(a) Baseline case

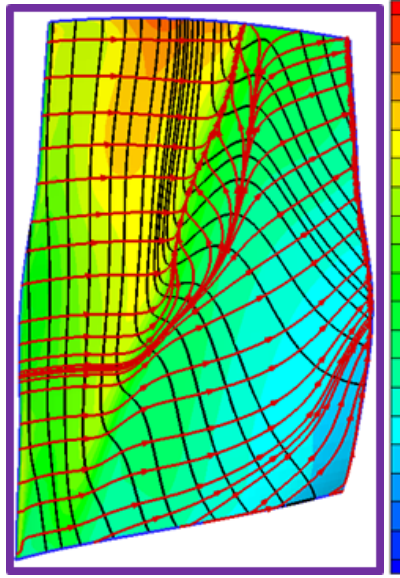

(b) Optimum case (1 \& 2)

Entropy $[\mathrm{J} /(\mathrm{kg} . \mathrm{k})]$

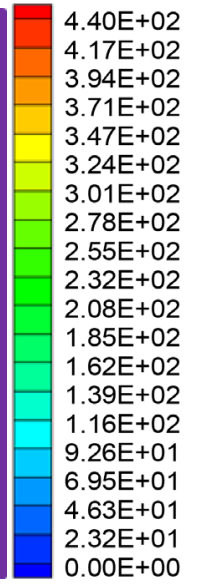

$0.00 \mathrm{E}+00$

Figure 25. Vector lines of $(\tau, \omega)$ and entropy on rotor blade suction side (black curve is $\omega$, red curve is $\tau$ ).

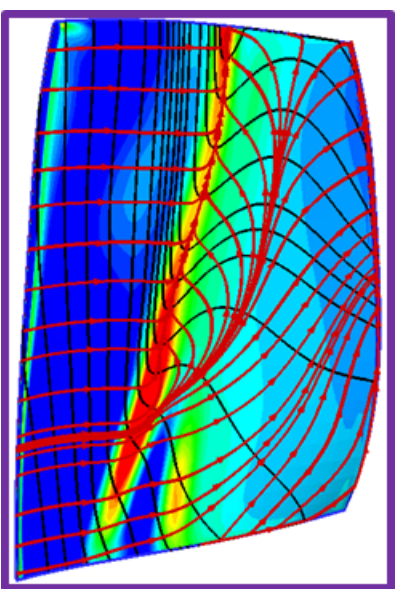

(a) Baseline case

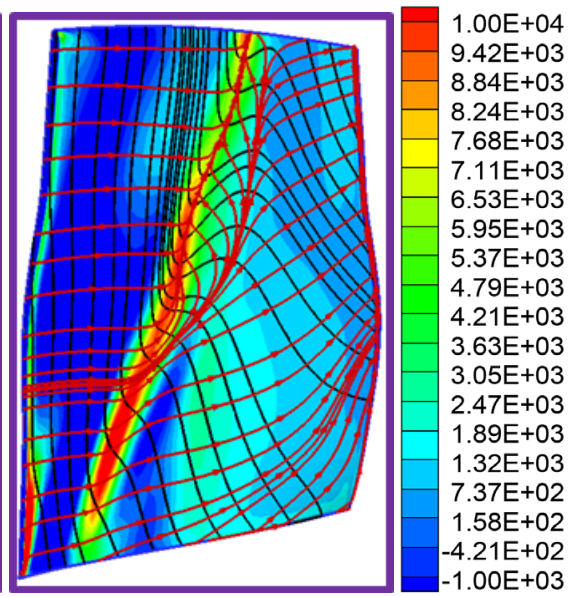

(b) Optimum case (1 \& 2)

Figure 26. Vector lines of $(\boldsymbol{\tau}, \boldsymbol{\omega})$ and axial gradient of entropy on rotor suction side (black curve is $\omega$, red curve is $\tau$ ).

immediately following the shock wave are weakened considerably, while the secondary flow is alleviated by the modification of the curvature on the airfoil camber line. Moreover, the sign of radial vorticity $\omega_{r}$ is reversed across the separation bubble. The beginning of the separation bubble has higher negative $\omega_{r}$ The start and end of the separation bubble coincide with the sign change of radial vorticity $\omega_{r}$ on the transonic rotor suction side.

The Boundary Vorticity Flux (BVF) is a very important vorticity dynamics parameter since it directly relates to the on-wall physical generation of vorticity [17] [18], and it was first defined by Lighthill in 1963 [31]. In general, on an accelerating body surface, applying the tangential components of $\mathrm{N}-\mathrm{S}$ equation to the surface indicates that the BVF consists of the contributions of the wall acceleration $a$, body force $f$, on-wall tangent pressure gradient, and a three-dimensional viscous correction as follows [21]: 


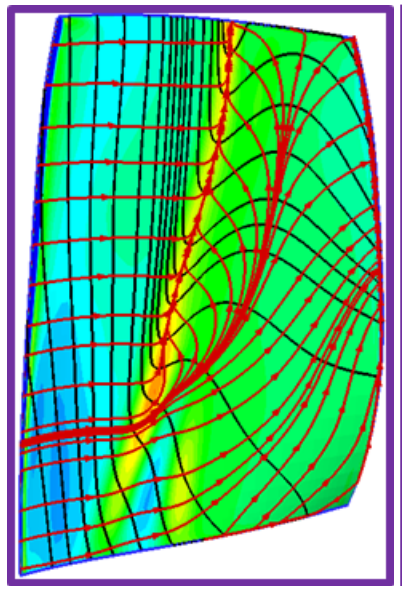

(a) Baseline case

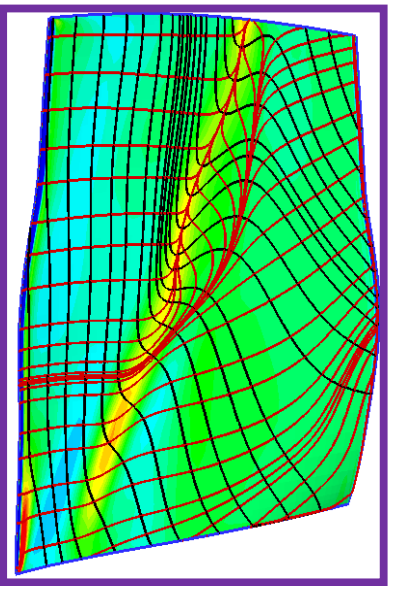

(b) Optimum case ( 1 \& 2)

$\mathrm{ds} / \mathrm{dz}[\mathrm{J} /(\mathrm{kg} \cdot \mathrm{k} \cdot \mathrm{m})]$

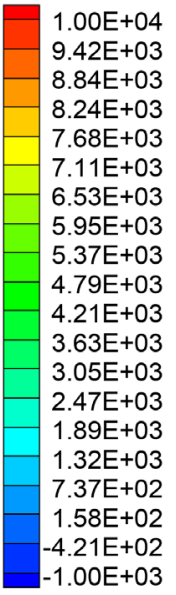

Figure 27. Vector lines of $(\boldsymbol{\tau}, \boldsymbol{\omega})$ and axial gradient of static pressure on rotor suction side (black curve is $\omega$, red curve is $\tau$ ).

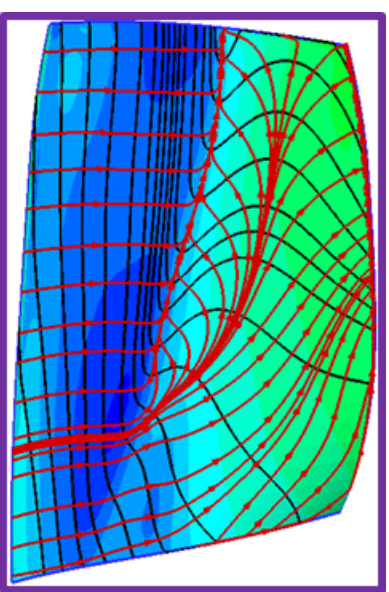

(a) Baseline case

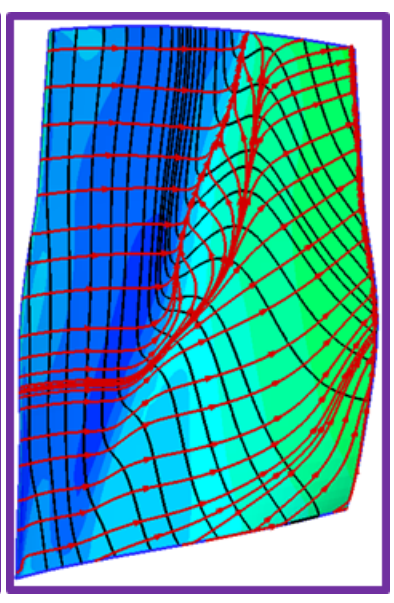

(b) Optimum case (1 \& 2)

Static pressure [pa]

Figure 28. Vector lines of $(\boldsymbol{\tau}, \boldsymbol{\omega})$ and static pressure on rotor suction side (black curve is $\omega$, red curve is $\tau$ ).

$$
\boldsymbol{\sigma}=\frac{\mu}{\rho} \boldsymbol{n} \cdot \nabla \boldsymbol{\omega}=v \frac{\partial \boldsymbol{\omega}}{\partial n}=\sigma_{a}+\sigma_{f}+\sigma_{p}+\sigma_{v i s}
$$

where,

$$
\begin{aligned}
& \boldsymbol{\sigma}_{\boldsymbol{a}}=\boldsymbol{n} \times \boldsymbol{a}_{B}, \boldsymbol{\sigma}_{f}=-\boldsymbol{n} \times \boldsymbol{f} \\
& \boldsymbol{\sigma}_{p}=\frac{\boldsymbol{n}}{\rho} \times[\nabla(p-\mu \nabla \cdot \boldsymbol{u})], \boldsymbol{\sigma}_{\text {vis }}=\frac{\mu}{\rho}(\boldsymbol{n} \times \nabla) \times \boldsymbol{\omega}
\end{aligned}
$$

where, $\boldsymbol{n}$ is unit normal vector for wall, $\boldsymbol{a}_{B}$ is wall accelerator. In general, for Newton fluid such as air, since the viscous coefficient with very small value and the body force is ignored, then $\boldsymbol{\sigma}_{p}=\frac{\boldsymbol{n}}{\rho} \times \nabla p$.

The axial component of $\sigma_{p}$ is denoted as $\sigma_{p z}$, and it is read in cylindrical coordinates system as follows: 


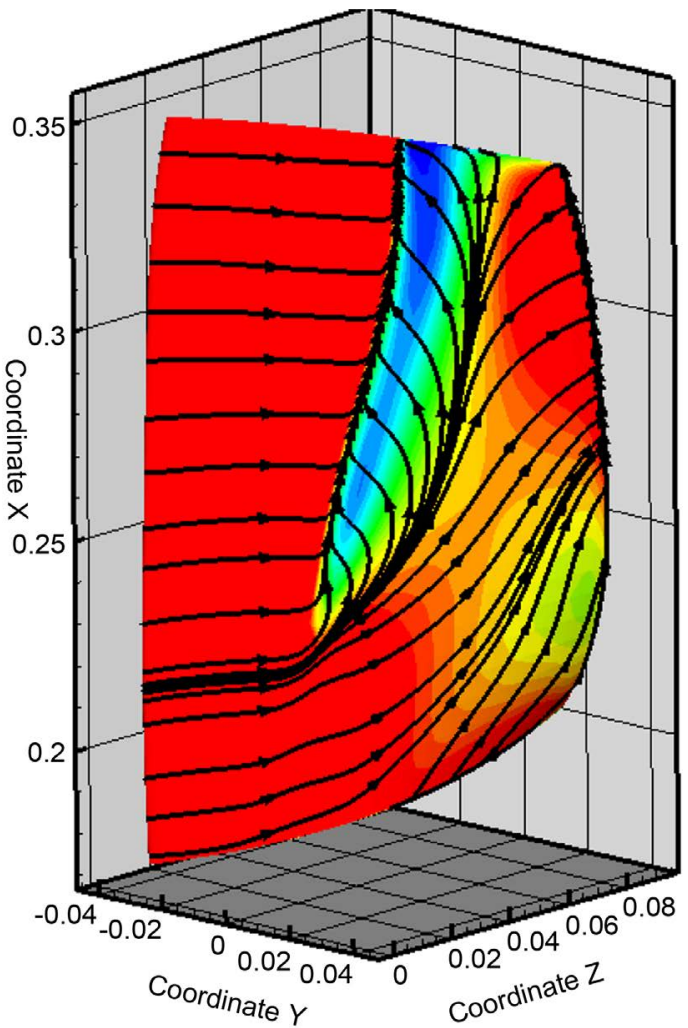

(a) Baseline case

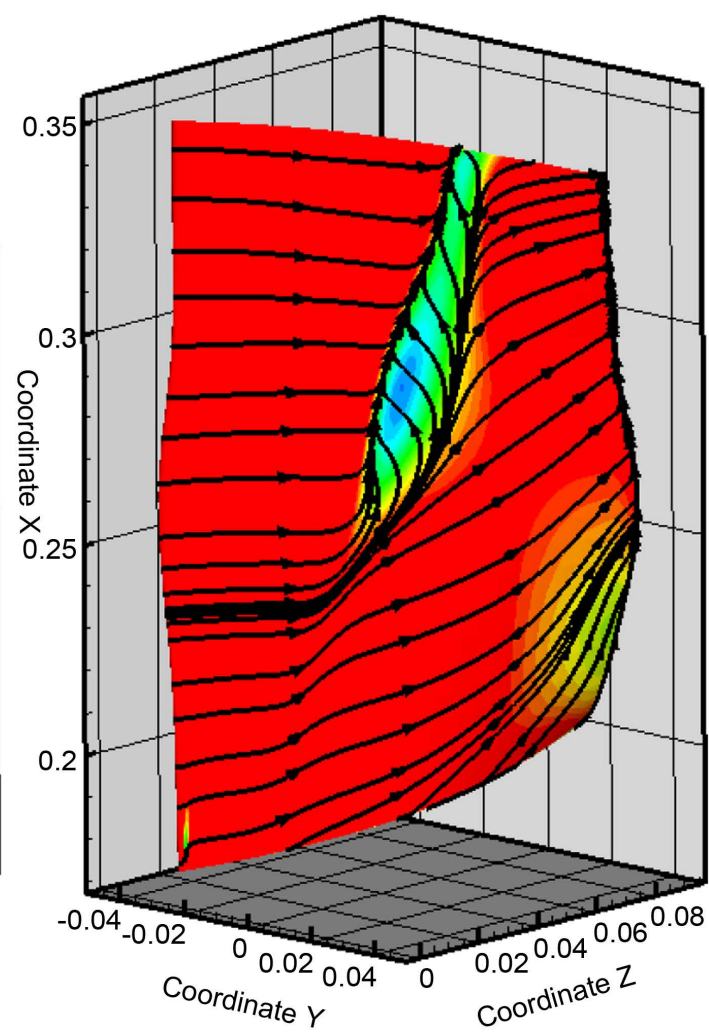

(b) Optimum case (1 \& 2)

Figure 29. 3D View of rotor 1 for Figure 19 and Figures 20-28.

$$
\sigma_{p z}=\frac{n_{r}}{r} \frac{\partial p}{\partial \theta}-n_{\theta} \frac{\partial_{p}}{\partial r}
$$

The literature [20] [21] indicates that the high $\sigma_{p z}$ positive peak is not beneficial due to a reduction in torque on the rotor blades. It mainly occurs near the shock on the suction side of a transonic compressor due to the strong compression effect as shown in Figure 20 for the baseline and optimum cases. It illustrates that the area of $\sigma_{p z}$ positive peak is further downstream for the optimum blade and shifted away from the sensitive flow regions, especially near the endwall region. Moreover, the shock position is postponed further downstream resulting in reduced shock strength to optimized rotor. Thus the gradient of pressure is alleviated and the flow loss is reduced for the optimized rotor. These effects are shown in Figure 21 (Static pressure on rotor suction side) and Figure 22 (Entropy on rotor suction side).

To better understand the boundary-layer separation on the rotor surface, a set of boundary-layer separation criterion has to be introduced from the literature [18], although these criteria may have some long-standing controversial issues. The three prime criteria on boundary-layer separation can be summarized as follows:

Separation zone warning: Not only the skin-friction vector $\tau$-lines converge, but also vorticity vector $\boldsymbol{\omega}$-lines signature on the wall have a large positive curvature, and BVF with a peak. 
Separation line criteria: The curvature of vorticity lines reach a maximum (Both for open and closed separation).

Separation watch: Tangent BVF $\sigma$-lines turn to the direction of skin-friction vector $\tau$-lines on wall, or the tangent pressure gradient vector lines are basically perpendicular to the separation line.

These boundary-layer separation criteria are sufficiently verified by vector lines of $(\tau, \boldsymbol{\omega})$ as shown in Figure 23 and vector lines of $\left(\tau, \sigma_{p}\right)$ as shown in Figure 24 on the rotor suction side. For the optimized rotor, the separation bubble radial and axial size are greatly reduced. Moreover, not only the hub corner secondary flow is abated, but also the separation line is further leaned to downstream near the shroud area, which means the shock wave is further downstream. Thus the shock strength is weakened moderately, and flow loss is decreased substantially. These flow features help explain why the optimized rotor has improved performance.

High entropy gradient and static pressure gradient is a necessary but not sufficient condition for boundary-layer separation as shown in Figure 26 and Figure 27. There must be a high gradient of entropy and static pressure for maximum positive on-wall signature along each $\boldsymbol{\omega}$-line. It may be the highest gradient of many flow parameters of a transonic rotor. Figures 25-28 illustrate that the derivative/gradient of entropy and static pressure are more effective than the quantity itself for transonic rotor flow field diagnosis. In contrast, there is smaller axial gradient on the suction surface for the optimum than that for baseline case, especially in upper half span.

\section{A 2.9 Pressure Ratio Transonic Fan Rotor Optimized Using Vorticity Dynamics}

\subsection{Optimization Methodology}

The axial moment acted on the fluid by the rotor blades due to pressure is:

$$
M_{z}=-\int_{S}(\boldsymbol{r} \times \boldsymbol{n} p)_{z} \mathrm{~d} S
$$

which is the torque due to pressure forces. The power is the torque times the angular rotation rate. Using the transformation

$$
(\boldsymbol{n} \times \nabla)\left(p r^{2}\right)=r^{2}(\boldsymbol{n} \times \nabla p)+2 p \boldsymbol{n} \times \boldsymbol{r}=\rho r^{2} \boldsymbol{\sigma}_{p}-2 \boldsymbol{r} \times(\boldsymbol{n} p)
$$

and Gauss theorem and Stokes theorem, formula (6) can be expressed as:

$$
M_{z}=-\int_{S}(\boldsymbol{r} \times \boldsymbol{n} p)_{z} d S=-\frac{1}{2} \int_{S_{b}} \rho r^{2} \sigma_{p z} d S+\frac{1}{2} \oint_{\partial S_{b}} p r^{2} d z
$$

Here, $S_{b}$ denotes rotor blade surface and $\partial S_{b}$ is the boundary of $S_{b}, \boldsymbol{M}$ is total moment vector of acting onto the fluid by the rotor blades, and $M_{z}$ is its axial-component. $\sigma_{p z}$ is axial-component of BVF $\sigma_{p}$. Equation (8) indicates that $M_{z}$ contains two parts, of which the first part is surface integration of the second-moment of $\sigma_{p z}$, while the second part is curve integration of the second-moment of static pressure $p$. Hence, it could be deduced that the lower $\sigma_{p z}$ is, the higher $M_{z}$ will be, through which more beneficial effects would be 
brought to the pressure rise capability of the rotor blade passage. Therefore, the axial component of BVF $\sigma_{p z}$ is an important parameter that reflects the fan/compressor aerodynamic performance. An interpretation of this new vorticity-based method is presented using the axial moment $M_{z}$ (torque due to pressure forces) as the objective function for optimization of the rotor blade in a ansonic fan. By maximizing the axial moment due only to pressure and not viscous forces is related to the efficiency since this torque is part of the efficiency equation that does useful work. The design parameters are shown in Table 3 and the optimization statements are as follows:

Maximize: axial-moment $M_{z}$ at the design point for fan rotor blade passage domain;

Constraints: mass flux $\dot{m} \geq \dot{m}$ (baseline), and total pressure ratio $\pi \geq \pi$ (baseline);

Design Variables: 20 variables for 4 sections, which have 3 points used to control curvature for the unit chord;

Genetic Algorithm (GA) parameters: population size is 12, and the maximum iteration is 300 ;

Constants: position and metal angle at leading edge and trailing edge for rotor in the meridian plane.

The baseline case of the fan rotor has been designed by a Turbomachinery Axisymmetric design code [28] (known as T-Axi), while the blade geometry is generated by $3 \mathrm{D}$ BGB [25]. Gridding topology is a best practice established in Autogrid. Total number of mesh cell is approximately 700,000 with $y+<1$ for first cell from the wall in order to capture flow details in the boundary-layer area. The S-A turbulence model has been used.

\subsection{Optimization Results and Discussion}

Table 4 displays a comparison of the aerodynamic performance for the optimum and baseline cases. With the total pressure ratio being 2.896 and the adiabatic efficiency reaching $88.49 \%$ for the optimum case, the stall margin of the fan rotor is still higher than $10 \%$ (up to $10.74 \%$ ), which is an inspiring result. Moreover, based on the performance map at the design speed line shown in Figure 30,

Table 3. Design parameters of a fan rotor.

\begin{tabular}{cc} 
Design parameters & Values \\
\hline $\begin{array}{cc}\text { Corrected first rotor tip tangent speed }(\mathrm{m} / \mathrm{s}) \\
\text { Corrected design angular velocity }(\mathrm{rpm})\end{array}$ & 495.32 \\
Design flow rate $(\mathrm{kg} / \mathrm{s})$ & $21,500.0$ \\
Tip flow coefficient of the rotor & 24.30 \\
Tip work coefficient of the rotor & 0.285 \\
Relative Mach Number at LE of rotor tip & 0.450 \\
Hub/tip ratio at the inlet of the rotor & 1.57 \\
The number of rotor blade & 0.41 \\
Clearance at rotor tip (mm) & 14 \\
\hline
\end{tabular}


the optimized highly-loaded fan rotor has a wide operating range with high adiabatic efficiency and favorable through flow capability along the whole operating line. This aerodynamic performance level is beyond the design of conventional axial fan rotor. Obviously, the optimization based on vorticity dynamics is one of promising novel design concepts.

Figure 31 shows the distribution of adiabatic efficiency and total pressure ratio profiles as a function of relative blade height. Compared with the baseline case, the total pressure ratio and adiabatic efficiency of the optimizated rotor are improved significantly from $20 \%$ span to $70 \%$ span. This is mainly attributed to the shift of the shock wave aft toward the trailing edge. Both the strength of the shock wave and the pressure gradient after the shock are reduced, as well as further control of the boundary layer. From $70 \%$ span to the tip area, the total pressure ratio is reduced slightly while the adiabatic efficiency is improved

Table 4. Comparison of results with baseline at design point.

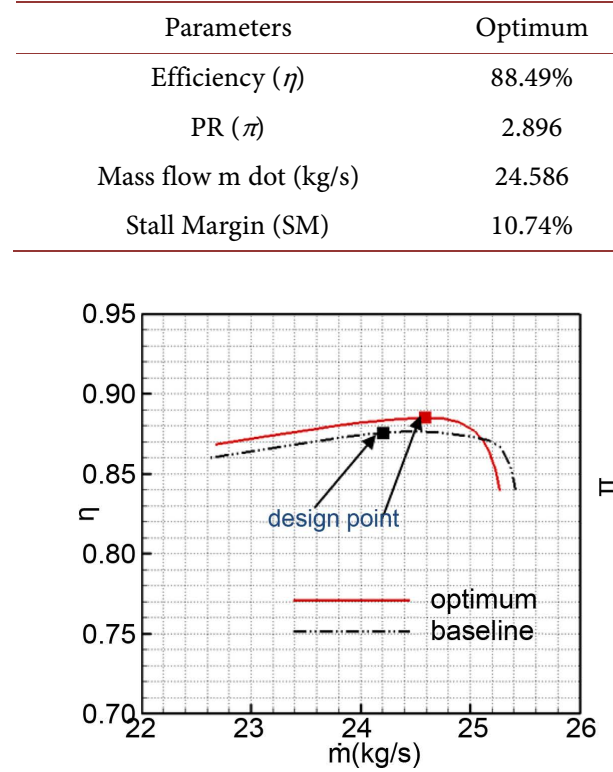

(a) Adiabatic efficiency

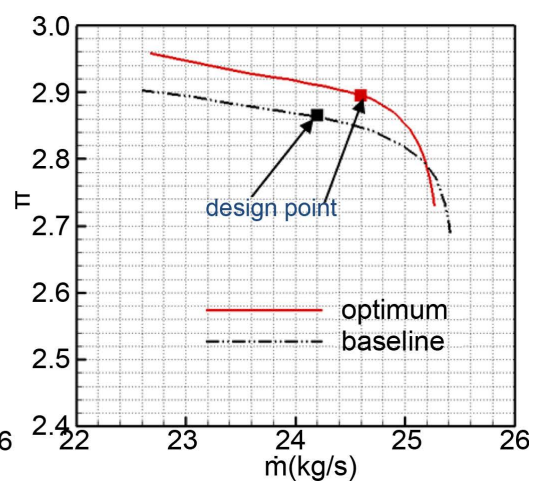

(b) Total pressure ratio

Figure 30. Performance map for the fan rotor at design speed line.

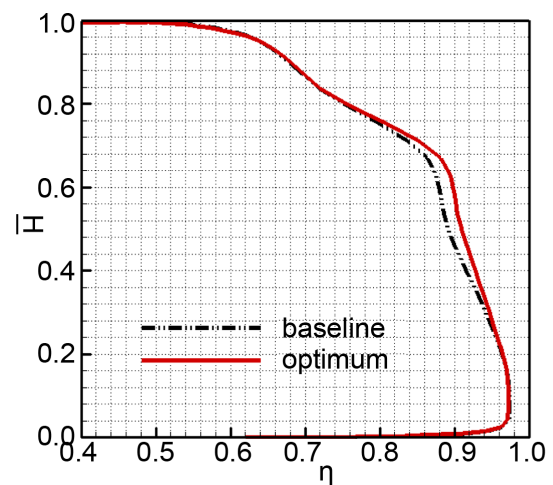

(a) Adiabatic efficiency

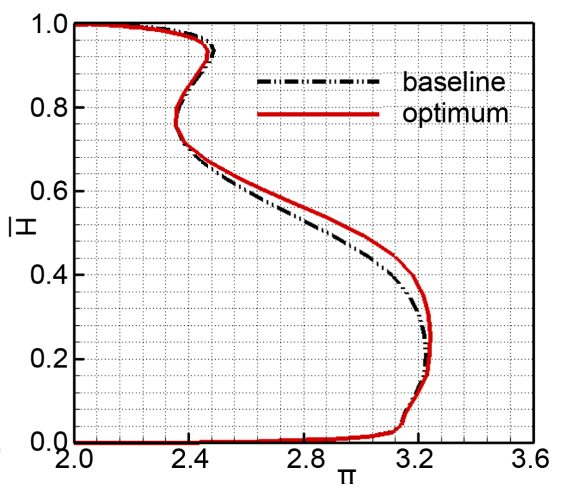

(b) Total pressure ratio

Figure 31. Comparisons of performance parameters for the rotor blade at design point. 
slightly due to high Mach Number in this area, so the optimization goal is to further control shock wave and secondary flow after the shock wave.

Figure 32 shows the BVF- $\sigma_{p z}$ and skin-friction vector $\tau$-lines on the rotor blade suction side at design point. As the figure shows, the positive peak of BVF in the upper half of the blade has a smaller area after the optimization. Meanwhile, secondary flow and shock wave are also weaker in the optimum case, which is beneficial to increase the adiabatic efficiency. These positive effects show that the control of camber line curvature is quite helpful to manage the shock wave and boundary layer flow. Also, the objective function based on vorticity dynamics $M_{z}$ is beneficial to increase total pressure ratio for the transonic fan rotor.

The Mach Number at 95\% span for the design point are shown in Figure 33 for both the baseline and optimized blades. The peak Mach Number is reduced for the optimized blade that yields an efficiency improvement. Figure 34 shows the Mach Number at $95 \%$ span at near stall also for both the baseline and optimized blade. Again the peak Mach Number is reduced for the optimized blade.

The stacked sections for the optimized blade are shown in Figure 35 while Figure 36 shows the $3 \mathrm{D}$ view of the optimized rotor design.

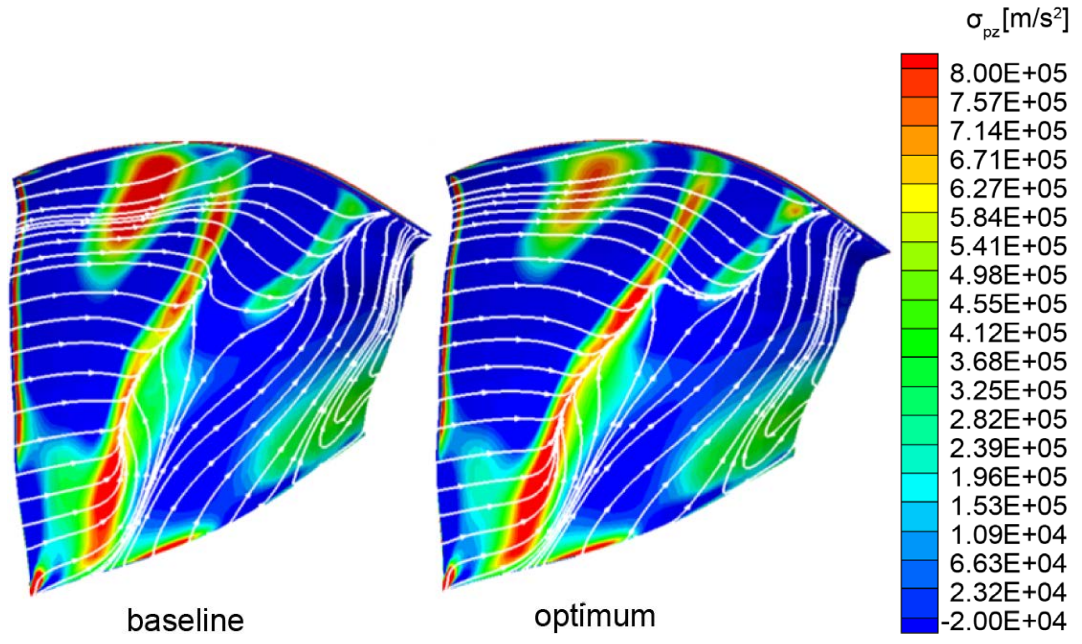

Figure 32. Comparisons of BVF- $\sigma_{p z}$ and skin-friction vector $\tau$-lines on the rotor blade suction side at design point.

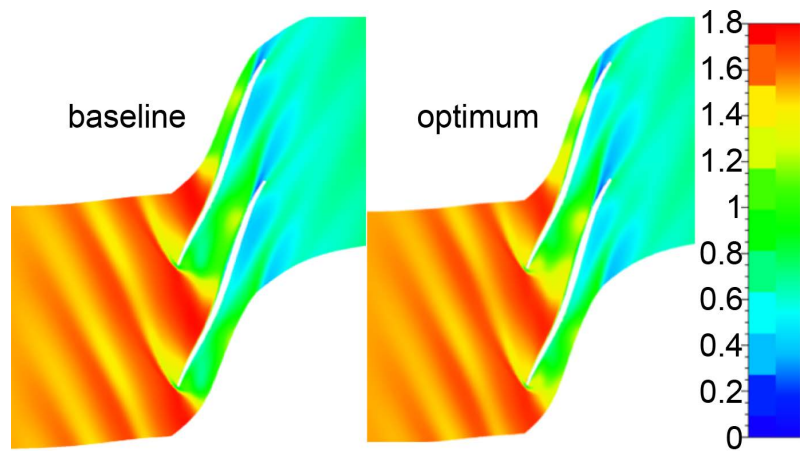

Figure 33. Mach Number at $95 \%$ span at the design point. 


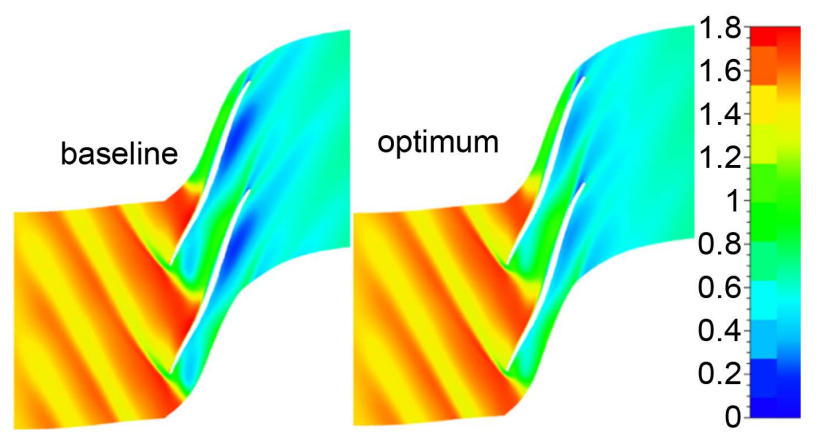

Figure 34. Mach Number at $95 \%$ span at near stall.

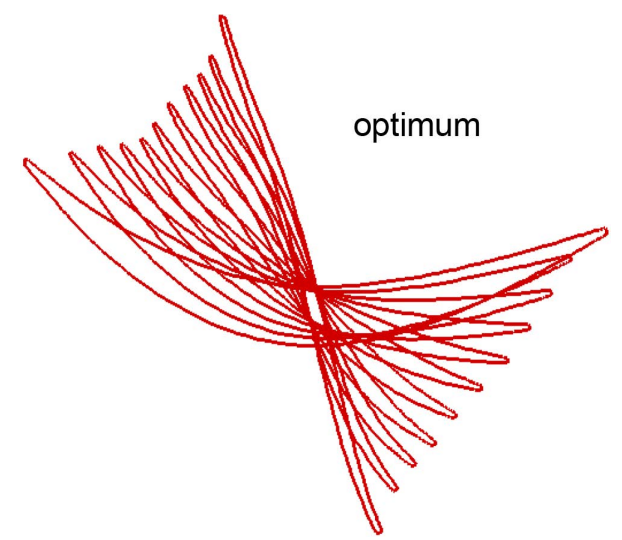

Figure 35. Sections stacked at centroid for optimized blade.

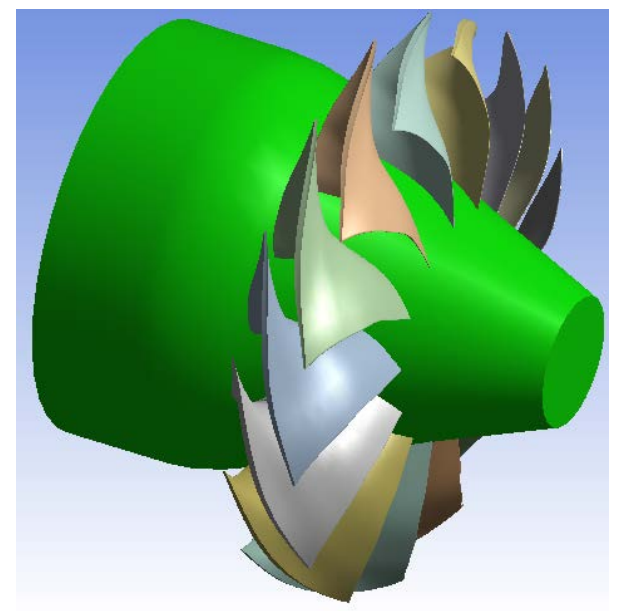

Figure 36. 3D view of optimized blade.

\section{Conclusions \& Future Work}

An optimization process has been implemented for two different transonic fans. The first optimization is the first stage plus IGV of a high pressure compressor base on the $\mathrm{GE} \mathrm{E}^{3}$ design. Only the rotor was modified while keeping the IGV and stator 1 unchanged. The leading edge and trailing edge flow angles in addition to the curvature of the rotor meanline up and down the span were allowed to vary in the optimization process. The parametric geometry generator is connected to a 3D CFD solver and a Single Objective Function Genetic Algorithm 
was used to drive the optimization. The optimization leads to an increase of adiabatic efficiency of $1.45 \%$ with a slight increase in total pressure ratio of $0.206 \%$. Furthermore, the flow rate is increased by $0.552 \%$ and stall flow rate is reduced by $1.541 \%$ which greatly improve stall margin and operation range. Improved stall margin was not stated goal of the optimization, and its improvement is attributed to simulating all three blade rows at once. Solving all three blade rows at the same time ensures that the rotor is being optimized within a multistage influence. In addition, the use of curvature of the meanline and ensuring smoothness spanwise of these parameters allows a small number of parameters to drive aerodynamically appropriate geometry changes through the optimization process.

In addition to traditional spanwise profiles and Mach Number explanations, a vortex/vorticity dynamics technique was used to further understand the improvements in performance. The results indicate that the rotor loading is restructured; the shock and the separated flow behind it together with the corner secondary flow on the rotor suction side have improved with the optimized blade.

The azimuthal vorticity and boundary vorticity flux (BVF) are two important parameters for which the utility has been demonstrated in this paper. The radial vorticity reverses sign on either side of the separation bubble generated by shock on the suction side. The "signature" of the separation bubble with high negative values of radial vorticity is captured with this approach. In addition, the axial derivate of entropy is high and is another significant feature of the transonic compressor rotor flow.

Comparing with the flow parameters themselves, checking their derivation/ gradient is more meaningful for the flow diagnosis, especially for the diagnosis of "focus flow", because the derivation/gradient for flow parameters can give abundant and crucial flow information for guiding designers to improve the turbomachinery performance.

A second optimization method based on vorticity dynamics theory has also been presented for a 2.9 Pressure Ratio Transonic Fan Rotor. For this case the objective function was the axial moment based on BVF. The results indicate that camber curvature control technology is very helpful to manage shock wave and boundary layer flow. The beneficial effect of the objective function $M_{z}$ based on vorticity dynamics in increasing the total pressure for transonic fan rotor is also verified. A rotor with $88.5 \%$ efficiency at 2.9 pressure ratio was produced. The optimization of turbomachinery through-flow geometry linked to vorticity dynamics may provide designers with novel prospects to further improve aerodynamic loading while enhancing the aerodynamic efficiency.

Optimization strategies are still evolving. It is clear that instead of taking the designer out of the loop, it allows the designers job to be one of deciding the objective function(s), constraints, and variables to modify. Understanding improvements is still required, and advanced methods such as vortex/vorticity dynamics become extremely useful. 
The present optimization study focused on improving efficiency based solely on modifying the rotor leading and trailing edge angles as well as controlling the curvature of the blade meanline sections. This was done to fully demonstrate the new capability for smooth curvature control and to allow a purely aerodynamic optimization since the blade stacking did not change nor did the area of each blade section. In addition a mixing plane CFD simulation was performed for the 3 blade row solutions, no fillet was modeled in the rotor, and the gaps under the IGV and stator 1 (they are both variable) were not modeled. Improved simulation to account for unsteadiness using harmonic methods and for more detailed geometry would be part of future work.

The current capability of the geometry generator includes smooth variations of the stacking axis to allow for lean and sweep as well as axial chord and thickness. Detailed leading edge designs are also possible. These geometry changes greatly affect the stress on a blade so a Finite Element stress analysis would have to be part of the optimization. This has been part of a multi-objective function optimization in another project where mass of the blade is the other objective function. Stall margin was not an explicit goal in the optimization, but did improve. Operability may have to be part of a formal optimization statement, and current efforts are exploring this. Some additional capability still needs to be added to the geometry generator. This includes non-axisymmetric endwalls using a method similar to the curvature control demonstrated in this paper. In addition, parametric cavity design is already being explored.

\section{Acknowledgements}

This work was supported by National Natural Science Foundation of China, Grant No. 51506036.

\section{References}

[1] Shang, E.B., Wang, Z.Q., et al. (1991) A Perspective and Effective Way to Improve the Compressor Cascade Performance. IGTC-13, Yokohama Int. Gas Turbine Congress.

[2] Deich, M.E., Gubalev, A.B., Filippov, G.A. and Wang, Z.Q. (1962) A New Method of Profiling the Guide Vane Cascade of Stage with Small Ratios Diameter to Length. Teplienergetika, 8, 42-46.

[3] Filippov, G.A. and Wang, Z.Q. (1963) The Calculation of Axial Symmetric Flow in an Turbine Stage with Small Ratio of Diameter to Blade Length. Journal of Moscow Power Institute, 47, 63-78.

[4] Wang, Z.Q., Su, J.X. and Zhong, J. (1994) The Effect of the Pressure Distribution in a Three-Dimensional Flow Field of a Cascade on the Type of Curved Blade. ASME 1994 International Gas Turbine and Aeroengine Congress and Exposition, Hague, 13-16 June 1994, No. 94-GT-400.

http://proceedings.asmedigitalcollection.asme.org/proceeding.aspx?articleid=21436 $\underline{38}$ https://doi.org/10.1115/94-gt-409

[5] Bliss, D.B., Hayden, R.P. and Murry, B.S. (1976) Design Considerations for Novel Low Source Noise Transonic Fan Stage. 3rd Aeroacoustics Conference, Palo Alto, 
20-22 July 1976, AIAA Paper No. 76-577. https://doi.org/10.2514/6.1976-577

[6] Lucas, J., Woodward, R. and Mackinnon, M. (1978) Acoustic Evaluation of a Novel Swept Rotor Fan. 11th Fluid and Plasma Dynamics Conference, Seattle, AIAA Paper No. 78-1121. https://doi.org/10.2514/6.1978-1121

[7] Wennerstrom, A.J. and Frost, G.R. (1976) Design of a 1500ft/sec, Transonic, Highthrough-Flow, Single-Stage Axial-Flow Compressor with Low Hub/Tip Ratio. Air Force Aero Propulsion Lab, Wright-Patterson AFB, No. AFARL-TR-76-59.

[8] Frank, B.J. and King, P.I. (1994) Effects of Leading Edge Sweep on Stall Inception in a High-Speed Single-Stage Compressor. 30 th Joint Propulsion Conference and EXhibit, Joint Propulsion Conferences, AIAA Paper No. 94-2696. https://doi.org/10.2514/6.1994-2696

[9] Boger, K.M., King, P.I. and Copenhaver, W.W. (1993) Stall Inception in Single Stage High-Speed Compressor with Straight and Swept Leading Edges. 29th Joint Propulsion Conference and Exhibit, Joint Propulsion Conferences, AIAA 93-1870.

[10] Benini, E. (2004) Three-Dimensional Multi-Objective Design Optimization of a Transonic Compressor Rotor. Journal of Propulsion and Power, 20, 559-565. https://www.researchgate.net/publication/235642689_Three-Dimensional_Multi-O bjective_Design_Optimization_of_a_Transonic_Compressor_Rotor https://doi.org/10.2514/1.2703

[11] Jang, C.M., Li, P. and Kim, K.Y. (2005) Optimization of Blade Sweep in a transonic Axial Compressor Rotor. JSME International Journal Series B, 48, 793-801.

https://www.researchgate.net/publication/245396662_Optimization_of_Blade_Swee p_in_a_Transonic_Axial_Compressor_Rotor https://doi.org/10.1299/jsmeb.48.793

[12] Lian, Y. and Liou, M.S. (2005) Multi-Objective Optimization of Transonic Compressor Blade Using Evolutionary Algorithm. Journal of Propulsion and Power, 21, 979-987. https://doi.org/10.2514/1.14667

[13] Ellbrant, L., Eriksson, L.E. and Martensson, H. (2013) Balancing Efficiency and Stability in the Design of Transonic Compressor Stages. Proceedings of ASME Turbo Expo 2013: Turbine Technical Conference and Exposition, San Antonio, 3-7 June 2013, GT2013-94838.

http://proceedings.asmedigitalcollection.asme.org/proceeding.aspx?articleid=17765 64\&resultClick=3

[14] Siddappaji, K., Turner, M.G. and Merchant, A. (2012) General Capability of Parametric 3D Blade Design Tool for Turbomachinery. Proceedings of ASME Turbo Expo 2012, Copenhagen, 11-15 June 2012, GT2012-69756.

http://proceedings.asmedigitalcollection.asme.org/proceeding.aspx?articleid $=16950$ 55\&resultClick $=3$

[15] Nemnem, A.F., Turner, M.G., Siddappaji, K. and Galbraith, M. (2014) A Smooth Curvature-Defined Meanline Section Option for a General Turbomachinery Geometry Generator. Proceedings of ASME Turbo Expo 2014: Turbine Technical Conference and Exposition, Düsseldorf, 16-20 June 2014, GT2014-26363.

http://proceedings.asmedigitalcollection.asme.org/proceeding.aspx?articleid=19075 06\&resultClick $=3$

[16] Nemnem, A.F. (2014) A General Multidisciplinary Turbomachinery Design Optimization System Applied to a Transonic Fan. Ph.D. Thesis, University of Cincinnati, Cincinnati.

[17] Wu, J.Z., Ma, H.Y. and Zhou, M.D. (2006) Vorticity and Vortex Dynamics. Springer Science \& Business Media, New York, Printed in Germany. https://doi.org/10.1007/978-3-540-29028-5 
[18] Wu, J.Z., Tramel, R.W., Zhu, F.L., et al. (2000) A Vorticity Dynamics Theory of Three-Dimensional Flow Separation. Physics of Fluids (1994-Present), 12, 19321954. http://aip.scitation.org/doi/abs/10.1063/1.870442 https://doi.org/10.1063/1.870442

[19] Wu, J.Z., Lu, X.Y., Yang, Y.T., et al. (2010) Vorticity Dynamics in Complex Flow Diagnosis and Management. Proceedings of the 13 th Asian Congress of Fluid Mechanics, Dhaka, 17-21 December 2010, 1-22.

https://www.researchgate.net/publication/266891986_Vorticity_Dynamics_in_Com plex_Flow_Diagnosis_and_Management

[20] Yang, Y., Wu, H., Li, Q., et al. (2008) Vorticity Dynamics in Axial Compressor Flow Diagnosis and Design. Journal of Fluids Engineering, 130, 041102.

http://fluidsengineering.asmedigitalcollection.asme.org/article.aspx?articleid=14781 $\underline{10}$ https://doi.org/10.1115/1.2903814

[21] Li, Q., Wu, H., Guo, M., et al. (2010) Vorticity Dynamics in Axial Compressor Flow Diagnosis and Design-Part II: Methodology and Application of Boundary Vorticity Flux. Journal of Fluids Engineering, 132, 011102. http://meetings.aps.org/Meeting/DFD07/Event/72001 https://doi.org/10.1115/1.4000650

[22] Fox, R.W., McDonald, A.T. and Pritchard, P.J. (2004) Introduce to Fluid Mechanics. 6th Edition, John Wiley \& Sons, Inc., Hoboken.

[23] Chen, H.L. and Turner, M.G. (2016) Vorticity Dynamics Based Flow Diagnosis for a 1.5-Stage High Pressure Compressor with an Optimized Transonic Rotor. ASME Turbo Expo 2016: Turbomachinery Technical Conference and Exposition, Seoul, 13-17 June 2016, GT-56682.

http://proceedings.asmedigitalcollection.asme.org/proceeding.aspx?articleid=25546 33\&resultClick $=1$

[24] Dakota. https://dakota.sandia.gov/

[25] 3DBGB. gtsl.ase.uc.edu/3DBGB

[26] Fine/Turbo. http://www.numeca.com/en/products/fineturbo

[27] Holloway, P., Knight, G., Koch, C. and Shaffer, S. (1982) Energy Efficient Engine High Pressure Compressor Detail Design Report. General Electric Company, Tech. Rep., NASA CR-165558.

[28] Turner, M.G., Merchant, A. and Bruna, D. (2011) A Turbomachinery Design Tool for Teaching Design Concepts for Axial-Flow Fans, Compressors, and Turbines. Journal of Turbomachinery, 133. http://www.asme.org/about-asme/terms-of-use https://doi.org/10.1115/1.4001240

[29] Mahmood, S.-H., Turner, M.G., Siddappaji, K. and Balasubramanian, K. (2016) Flow Characteristics of an Optimized Axial Compressor Rotor Using Smooth Design Parameters. Proceedings of ASME Turbo Expo 2016: Turbomachinery Technical Conference and Exposition, Seoul, GT2016-57028.

http://proceedings.asmedigitalcollection.asme.org/solr/searchresults.aspx?q=Flow\% 20Characteristics\%20of\%20an\%20Optimized\%20Axial\%20Compressor\%20Rotor\% 20using\%20Smooth\%20Design\%20Parameters\&f_ContentType=Proceedings\&Sear chSourceType $=3$ https://doi.org/10.1115/gt2016-57028

[30] Tecplot/User's Manual. http://www.tecplot.com/

[31] Lighthill, M.J. (1963) Introduction: Boundary Layer Theory: Laminar Boundary Layer. Oxford University Press, Oxford, 46113. 


\section{Nomenclatures}

\begin{tabular}{|c|c|}
\hline$A$ & Area or zone $\left(\mathrm{m}^{2}\right)$ \\
\hline$(r, \theta, z)$ & $\begin{array}{l}\text { Cylindrical coordinates with the axial } z \text {-direction; when used as } \\
\text { subscripts denotes the corresponding components of a vector }\end{array}$ \\
\hline$(x, y)$ & 2D Cartesian coordinates for general curve \\
\hline$y^{\prime}=\mathrm{d} y / \mathrm{d} x$ & The first derivative for $y$ about $x$ \\
\hline $\overrightarrow{y^{\prime \prime}}=\mathrm{d} \overrightarrow{y^{\prime}} / \mathrm{d} x$ & The second derivative for $y$ about $x$ \\
\hline$C$ & Curvature for general 2D curve \\
\hline$R$ & Radius of Curvature for general 2D curve \\
\hline$(s, n)$ & $\begin{array}{l}\text { Curve coordinates for curve coordinates system; when used as } \\
\text { subscripts denote corresponding components }\end{array}$ \\
\hline$p$ & Static pressure on fluid (pa) \\
\hline $\boldsymbol{u}$ & Velocity vector on fluid in inertial reference frame $(\mathrm{m} / \mathrm{s})$ \\
\hline $\boldsymbol{w}$ & Velocity vector on fluid in relative reference frame $(\mathrm{m} / \mathrm{s})$ \\
\hline$(u, v)$ & Non-dimensional coordinates in basic airfoil plane \\
\hline$m^{\prime}$ & Normal coordinate for the streamline \\
\hline$P R$ & Total pressure ratio on compressor \\
\hline $\mathrm{d} p / \mathrm{d} z$ & Axial gradient of static pressure for fluid \\
\hline $\mathrm{d} s / \mathrm{d} z$ & Axial gradient of static entropy for fluid \\
\hline $\bar{H}$ & $\begin{array}{l}\text { Relative height for blade ( } 0 \text { - for hub position, } 1 \text { - for shroud po- } \\
\text { sition) }\end{array}$ \\
\hline$\dot{m}$ & Mass flow rate for compressor/fan $(\mathrm{kg} / \mathrm{s})$ \\
\hline Ma or $M$ & Local Mach Number on fluid \\
\hline$M_{z}$ & Axial Moment due to pressure (N.m) \\
\hline$c$ & Local meridian curve distance $(\mathrm{m})$ \\
\hline$C_{m}$ & Total meridian curve distance $(\mathrm{m})$ \\
\hline inc & $\Delta \beta$ at the leading edge of the blade $\left({ }^{\circ}\right)$ \\
\hline dev & $\Delta \beta$ at the trailing edge of the blade $\left({ }^{\circ}\right)$ \\
\hline$c p_{1}$ & Control point at leading edge for rotor \\
\hline$c p_{t}$ & Control point at trailing edge for rotor \\
\hline
\end{tabular}

\section{Greek Symbols}

$\begin{array}{ll}\rho & \text { Density on fluid }\left(\mathrm{kg} / \mathrm{m}^{3}\right) \\ \pi & \text { Total pressure ratio on compressor } \\ \eta & \text { Adiabatic efficiency on compressor } \\ \tau & \text { Skin-friction vector for fluid (Fluid to wall) }\left(\mathrm{m}^{3} / \mathrm{s}^{2}\right) \\ \omega & \text { Vorticity vector of fluid (1/s) } \\ \sigma_{p} & \text { Boundary vorticity flux (BVF) vector by static pressure gradient } \\ & \left.\text { on wall (m/ } / \mathrm{s}^{2}\right) \\ \sigma_{p z} & \text { Axial component of } \sigma_{p} \\ \Omega & \text { Angular velocity of compressor rotor }(\mathrm{rpm}) \\ \Delta \beta & \text { Deviation on metal angle }\left({ }^{\circ}\right) \\ \Gamma=r u_{q} & \text { Circulation }\left(\mathrm{m}^{2} / \mathrm{s}\right)\end{array}$




\section{Subscripts}

$\begin{array}{ll}m & \text { Meridional plane } \\ \text { is } & \text { Isentropic } \\ f & \text { Body force for fluid per unit mass }\left(\mathrm{m}^{2} / \mathrm{s}^{2}\right)\end{array}$

Submit or recommend next manuscript to SCIRP and we will provide best service for you:

Accepting pre-submission inquiries through Email, Facebook, LinkedIn, Twitter, etc. A wide selection of journals (inclusive of 9 subjects, more than 200 journals)

Providing 24-hour high-quality service

User-friendly online submission system

Fair and swift peer-review system

Efficient typesetting and proofreading procedure

Display of the result of downloads and visits, as well as the number of cited articles Maximum dissemination of your research work

Submit your manuscript at: http://papersubmission.scirp.org/

Or contact ojfd@scirp.org 